\title{
A Tiered Approach to Systemic Toxicity Testing for Agricultural Chemical Safety Assessment
}

John E. Doe

Syngenta CTL, Macclesfield, United Kingdom

Alan R. Boobis

Imperial College London, United Kingdom

Ann Blacker

Bayer CropScience, Research Triangle Park, North Carolina, USA

Vicki Dellarco

U.S. Environmental Protection Agency, Office of Pesticide Programs, Washington, DC, USA

Nancy G. Doerrer

ILSI Health and Environmental Sciences Institute, Washington, DC, USA

\section{Claire Franklin}

Health Canada, Pest Management Regulatory Agency, Ottawa, Ontario, Canada

\section{Jay I. Goodman}

Michigan State University, East Lansing, Michigan, USA

\section{Joel M. Kronenberg}

Monsanto Company, St. Louis, Missouri, USA

\section{Richard Lewis}

Syngenta CTL, Macclesfield, United Kingdom

\section{Ernest E. McConnell}

Tox Path, Inc., Raleigh, North Carolina, USA

\section{Thierry Mercier}

INRA-GMPV-RD, Versailles, France

\section{Angelo Moretto}

Università di Padova, Padova, Italy

\section{Canice Nolan}

Delegation of the European Commission, Washington, DC, USA

\section{Stephanie Padilla}

U.S. Environmental Protection Agency, National Health and Environmental Effects Research Laboratory, Research Triangle Park, North Carolina, USA

The views expressed in this article are those of the authors and do not necessarily reflect the views or policies of the U.S. Environmental Protection Agency, the European Commission, or other institutions represented by the authors.

Claire Franklin is currently with The McLaughlin Centre for Population Health Risk Assessment, University of Ottawa, Ontario, Canada.

Lorraine Tilbury is currently with Pfizer Global Research \& Development, Amboise, France.

Address correspondence to Nancy G. Doerrer, ILSI Health and Environmental Sciences Institute, One Thomas Circle, NW, Ninth Floor, Washington, DC 20005, USA. E-mail: ndoerrer@hesiglobal.org 


\title{
Whang Phang
}

U.S. Environmental Protection Agency, Office of Pesticide Programs, Washington, DC, USA

\section{Roland Solecki}

Federal Institute for Risk Assessment, Berlin, Germany

\section{Lorraine Tilbury}

Dupont de Nemours Ag Products, Nambsheim, France

\section{Bennard van Ravenzwaay}

BASF Aktiengesellschaft, Ludwigshafen, Germany

\section{Douglas C. Wolf}

U.S. Environmental Protection Agency, National Health and Environmental Effects Research Laboratory, Research Triangle Park, North Carolina, USA

\begin{abstract}
A proposal has been developed by the Agricultural Chemical Safety Assessment (ACSA) Technical Committee of the ILSI Health and Environmental Sciences Institute (HESI) for an improved approach to assessing the safety of crop protection chemicals. The goal is to ensure that studies are scientifically appropriate and necessary without being redundant, and that tests emphasize toxicological endpoints and exposure durations that are relevant for risk assessment. The ACSA Systemic Toxicity Task Force proposes an approach to systemic toxicity testing as one part of the overall assessment of a compound's potential to cause adverse effects on health. The approach is designed to provide more relevant data for deriving reference doses for shorter time periods of human exposure, and includes fewer studies for deriving longer term reference doses-that is, neither a 12-month dog study nor a mouse carcinogenicity study is recommended. All available data, including toxicokinetics and metabolism data and life stages information, are taken into account. The proposed tiered testing approach has the potential to provide new risk assessment information for shorter human exposure durations while reducing the number of animals used and without compromising the sensitivity of the determination of longer term reference doses.
\end{abstract}

Keywords Agricultural Chemicals, Health-Based Guidance Values, Risk Assessment, SABRE Database, Systemic Toxicity Testing, Tiered Testing

\section{INTRODUCTION}

The ILSI Health and Environmental Sciences Institute (HESI) formed the Agricultural Chemical Safety Assessment (ACSA) Technical Committee in the year 2000 to design a toxicity testing scheme that would incorporate current understanding of pesticide toxicology and exposure and recognize the specificity of agricultural products. The purpose of and background for the ACSA project are described in detail in the companion paper by Carmichael et al. (2006).

As the proposed tiered testing approach for agricultural chemical safety assessment evolved, the ACSA Technical Committee and its task forces (Barton et al., 2006; Carmichael et al., 2006; Cooper et al., 2006) worked toward the following objectives:

- Provide information that can be applied to a range of relevant human exposure situations.

- Characterize effects that have the potential to damage human health at exposure levels approximating those that might be encountered in the use of these compounds.
- Avoid high doses that cause unnecessary public concern (e.g., safety assessments should focus on doses that are relevant to realistic human exposures while maintaining adequate power for the experimental studies to detect toxicity).

- Use the minimum number of animals necessary to produce a thorough safety assessment of the chemicals of interest.

- Inflict the minimum amount of distress on animals.

- Minimize excessive and unnecessary use of resources by regulatory authorities and industry which could be used to address other issues of concern.

- Increase both the efficiency and relevance of the current safety assessment process.

\section{Definition of the Scope of Systemic Toxicity}

The goal of the Systemic Toxicity Task Force (the Task Force) of the ACSA Technical Committee was to develop a hierarchy of study types, endpoints, and triggers for a decision tree for other 
toxicity endpoints, such as neurotoxicity, carcinogenicity, and chronic toxicity. The Task Force defined "systemic toxicity" for the purposes of the HESI project as the potential adverse effects of agricultural chemicals on "young adults." In this context, systemic toxicity includes general toxicology and specific effects on the nervous system, the immune system, and the endocrine system; genetic toxicology; carcinogenicity; and irritancy and sensitization.

The assessment of systemic toxicity in "young adults" is often the first step in the overall assessment of a compound's potential to cause adverse effects on health. The findings in young adults are then taken into account during the assessment of the compound during different life stages, including reproduction, development, adolescence, and the elderly. The process for assessing the effects of agricultural chemicals on these life stages is the subject of the companion paper by the ACSA Life Stages Task Force (Cooper et al., 2006). A complete assessment can be made only by taking into account all of the data generated in the overall assessment. Modes of action may be revealed in the assessment of systemic toxicity that will also reveal themselves in the assessment of different life stages. The assessments are underpinned by knowledge of toxicokinetics, from which internal, rather than externally applied, dose can be related to effects. The ACSA ADME Task Force has reviewed the assessment of toxicokinetics data (Barton et al., 2006). The work of all three ACSA Task Forces is complementary and should be considered together. A key aspect of the overall approach described here is to provide data that shed light on the shape of the dose-response curve, rather than emphasizing effects that may occur only at high doses. Reference doses are usually set on the critical effects, that is, the first effects occurring as the dose level is increased, although such dose levels are usually discovered by reducing the dose level until effects are no longer observed.

\section{ASSESSMENT OF THE CURRENT TESTING STRATEGY}

\section{Comparison of the Agricultural Chemical Testing Strategy with Safety Assessment Strategies for Other Chemicals}

The Task Force reviewed the way in which food additives, biocides, and pharmaceuticals are assessed by regulatory authorities in Europe and the United States. Each includes the concept that the studies that are done should reflect the toxicity of the chemical and its use profile.

The factors that are taken into account include:

- Exposure level, frequency, and duration.

- Level of toxicological concern about the chemical.

Each regulatory agency has a declared intention to limit the number of animals used in assessing the potential hazards of a chemical.

The testing schemes for food additives, biocides, and pharmaceuticals differ from the current agricultural chemical testing scheme in that they allow more opportunity for the use pro- file, the resulting exposure, and the toxicological profile of the chemical to influence the design of the evaluation program.

\section{Human Exposure Durations Required for Risk Assessments}

The range of required risk assessments has increased in recent years as knowledge has grown concerning the range of exposures to which humans may be subjected. Initially, the assessment of agricultural chemicals focused on continuous dietary exposure, which gave rise to the concept of the acceptable daily intake (ADI) for a lifetime. However, it is now recognized that there is a much broader range of exposure durations that must be considered, including exposures of workers applying agricultural chemicals, exposures of bystanders, home use, and short-term exposures in the diet. The Task Force identified the following human exposure durations for which risk assessments may be required:

- One day.

- Two to 28 days.

- One to 6 months.

- Greater than 6 months.

The Task Force considered whether there should be further division of the duration of exposure of "greater than 6 months," bearing in mind that this exposure duration ranges from 7 months of exposure to a lifetime's exposure, and that it would, therefore, be equivalent to the traditional lifetime ADI. The Task Force concluded that there would be relatively few occasions when a reference dose derived for a lifetime would be excessively conservative for 7 months exposure duration. Such situations would likely become apparent as the chemical properties are examined and the testing program progresses.

Exposure can occur from a variety of sources, including exposure to the concentrated compound during manufacture, transport, and dispensing; exposure to more dilute compound during the application of the compound; and exposure to residues in diet and drinking water. In addition, intermittent exposure should be considered. The Task Force created a data set that could provide risk assessors, who are acting to safeguard the health of those using agricultural chemicals or who may be exposed to them in diet, drinking water, or as bystanders, with information to allow the assessment of the impact of a wide range of potential human exposures.

\section{Matching Current Studies with Exposure Durations for Risk Assessments}

The current testing strategy employed by regulatory agencies around the world is primarily focused on two scenarios:

- Single exposure to large amounts of the undiluted substance.

- Prolonged exposure to the substance in the diet. 
In addition, there are two areas of concern for regulatory authorities that have received attention in recent years and for which new study designs have been or are under development:

- Medium-term exposure during mixing/loading by operators.

- Single-day dietary exposure.

The acute toxicity package of oral, dermal, inhalation studies, skin and eye irritation, and skin sensitization is designed to assess the effects of single exposures to the undiluted substance. The acute toxicity studies use death or severe toxicity as an endpoint, and the data are applied primarily for classification into various hazard categories for transport and general handling purposes.

The effect of prolonged exposure in the diet is assessed by the current program of studies that starts with a 28-day rat study. Note that the 28-day rat study is often viewed as a dose-setting study, and is not always submitted to regulatory authorities. The program of studies then proceeds to the 90-day rat study and the chronic rat study, which usually includes an assessment of carcinogenicity. In addition, a parallel series of studies is done in the dog (90-day and 1-year). A mouse carcinogenicity study is performed. Data are also generated to explore the effect of the chemical on reproduction and development. These studies have been considered by the ACSA Life Stages Task Force (Cooper et al., 2006). The data from these studies are used to identify and characterize the hazards of the substance, that is, what effects it causes and at which dose levels. This information is used in risk assessments. Over the three decades since this process was introduced, it has become apparent that it no longer meets all the needs of risk assessors who are acting to safeguard the health of those using agricultural chemicals or who may be exposed to them in diet, drinking water, or as bystanders. Concerns about the effects of agricultural chemicals have broadened from single, intermittent, and prolonged dietary exposure, to include inadvertent exposure in drinking water and application of chemicals in and around the home and other buildings. Despite these concerns, risk assessors must use the database provided by the current testing strategy and devise ways to extrapolate the data to meet their needs. A core principle of this project is to start with the needs of risk assessors and design a series of studies to meet those needs.

Focusing on risk assessment as the purpose for toxicology studies calls into question whether all the studies that are currently conducted are actually needed. The purpose of hazard characterization is to identify the likely adverse health effects, and provide a dose that, if not exceeded, would prevent these adverse health effects from occurring. This reference dose (RfD) can then be compared to the actual or projected exposure through the process of risk assessment. In most cases, when the database is complete using the large number of animals mandated by the test guidelines, only one study is used to set the RfD for each risk assessment. The question then arises: Would it have been possible to eliminate the studies which were not used for risk assessment and still protect human health?

Through the use of improved, expanded, and better designed studies, the Task Force proposes that it is possible to limit the number of animal studies required while still identifying the potential human health hazards of a chemical.

\section{ANALYSIS OF THE USE OF THE CURRENT DATABASE}

The current program for the assessment of agricultural chemicals has been in place for more than the last quarter of the 20th century. Although it has evolved somewhat during that time, its core has remained essentially unchanged. Hundreds of chemicals have been evaluated, which together form a rich database on the toxicity of agricultural chemicals. The Task Force acknowledges that any changes to the current assessment program must necessarily be based on sound scientific data. Consequently, a representative cross section of the large data set on the toxicity of agricultural chemicals was selected as a reference point for the Task Force. This data set was examined to determine which studies have been used most often to set the key reference RfDs for chemicals. The data guided the Task Force in the selection of study types to be used in the future and the identification of study types that may not be necessary. To achieve this objective, the Task Force commissioned the SABRE (Safety Assessment by Refined Experimentation) database described next.

\section{Description of the SABRE Database and Other Data Sources}

Initially, two to six chemicals from each class of pesticide (i.e., organophosphates, carbamates, pyrethroids, etc.) were randomly selected from a list of chemicals whose toxicology database had been evaluated by the Hazard Identification Assessment Review Committee (HIARC) of the Health Effects Division (HED), Office of Pesticide Programs (OPP), U.S. Environmental Protection Agency (EPA). During the U.S. EPA evaluation process, various toxicity endpoints (such as acute and chronic RfDs, incidental oral or dermal endpoints) were selected for risk assessment purposes. For each pesticide, the rationale for the toxicity endpoints and the conclusion of the peer review were recorded in a HIARC document, which served as a good starting point for data mining. The document contained all the necessary record keeping information in the OPP database (i.e., PC code and MRID number). With the PC code, the appropriate Registration Eligibility Document (RED) or Registration Standard could be easily accessed. In a RED, a summarized section of the toxicity for a pesticide is excerpted from the Toxicology Chapter prepared by HED. From the Toxicology Chapter, the individual study data evaluation report (DER) could be obtained. Within the Toxicology Chapter, a toxicology profile of a pesticide was always prepared and included. The toxicology profile of a pesticide contained brief summaries of essentially all the relevant toxicity studies used to establish toxicity endpoints. Information included date of the study, dose levels tested, no-observedadverse-effect level (NOAEL), lowest-observed-adverse-effect 
level (LOAEL), and the effects seen at the LOAEL. The toxicology profile was a crucial source for data mining. For the selected pesticide, another database, Toxicology Onliner, was also searched for additional information on older studies, if necessary. Under some circumstances, the HIARC report, Toxicology Chapter, and the toxicology profile did not provide a clear description of the effects; the DER of an individual study was always consulted.

For tumor incidences of carcinogenic pesticides, the Cancer Peer Review Document was consulted. For classification and the mode of pesticidal action for each pesticide, The Pesticide Book (Ware, 2000) was consulted.

The relevant toxicology information of each selected pesticide was extracted from the toxicology profile, HIARC report, Toxicology Onliner, DER, and The Pesticide Book (Ware, 2000) to be entered into the data spreadsheet. The information consisted of the summarized results of subchronic oral, dermal, and/or inhalation toxicity studies in rats and dogs; chronic toxicity studies in rats and dogs; carcinogenicity studies in rats and mice; developmental toxicity studies in rats and rabbits; multigeneration studies in rats; acute and subchronic neurotoxicity studies in rats; and any pertinent special studies. The LOAEL and NOAEL used to establish acute and chronic RfDs, tumor incidence, any sex difference in response to the chemical, target organ of toxicity, and the mode of toxicity (if demonstrated) were also entered into the database. In addition, the values for the acute and chronic RfDs were included. Toxicity information on 65 pesticides was entered into the database.

\section{Interrogation of SABRE and Other Databases}

The SABRE database, which was built specifically to assist the analyses presented here, allowed a unique series of questions to be answered. Of the 65 chemicals held in SABRE, 28 possessed appropriate data (from the core types of study required) for the current systemic toxicity testing paradigm for agricultural chemicals.

These core studies are the 90-day studies in the rat and dog, the combined chronic and carcinogenicity study in the rat, the 1-year study in the dog, and the carcinogenicity study in the mouse.

Using this data set, it was possible to address the following key questions concerning the study durations and test species currently used:

- The frequency with which the rat or the dog is used in regulation (specifically in setting the chronic RfD for a chemical).

- The identity of study types (durations and species) that are not used or that provide data that can be obtained from studies of a shorter duration or in a different species.

In addressing these questions using the SABRE database, a direct comparison may be drawn between the human durations relevant to risk assessment and the utility of current studies in risk assessment. Most importantly, the analyses were used to differentiate between those study types that should be retained and developed for future use, and those that should not.

\section{The Frequency with which the Rat or the Dog Is Used in Setting the Chronic RfD for a Chemical}

The purpose of using two species is to increase the probability of detecting an effect that would be potentially adverse to humans. The second species is used to cover the possibility that the rat may not be susceptible to an effect of a compound to which humans are susceptible, or the rat may be significantly less sensitive than humans to an effect. The use of a second species that is phylogenetically removed from the rat increases the likelihood that one or the other of the species will be at least as sensitive and/or susceptible as humans. In Table 1, data for 28 chemicals are summarized. These are the chemicals from the SABRE database for which there is a complete data set from 90day rat, 90-day dog, 24-month rat, and 1-year dog studies. The outcome of the key studies is provided, as is a brief description of the critical toxicity endpoints and the species used in risk assessment to set the chronic RfD for each chemical. The following conclusions may be drawn from analysis of the representative set of chemicals drawn from the sources cited already:

- For this set of chemicals, 14 out of $28(50 \%)$ are regulated on the outcome of studies in the dog. Twelve (43\%) of these chemicals showed increased sensitivity to the chemical- that is, the same effects were seen but at lower doses than in the rat. Two (7\%) showed a different susceptibility to the chemical-that is, the chemical caused different effects in the dog, which also had a lower NOAEL than the overall NOAEL in the rat derived from different effects. For one, the NOAEL was very high in both rat and dog, and the rat NOAEL would be adequately protective of the effect seen in the dog. For the other, the rat was more sensitive than the dog (at 90 days), and the rat NOAEL would again be adequately protective of the effect seen in the dog. These chemicals are identified in Table 1 by having either "sens" (sensitivity, same effect, lower dose) or "susc" (susceptibility, different effect) in the final column if the dog is the species setting the chronic RfD.

\section{The Identity of Study Types Not Used in Risk Assessment}

Studies in the Rat and the Dog. In comparing the outcome of studies of different duration in the rat, the relationship between the respective NOAELs can be variable. However, the NOAEL from the 90-day study is generally higher than the NOAEL in the 2-year study for the same toxicity endpoint, indicating that, for this species, the NOAEL can decrease with increasing period of exposure. For the dog, the quantitative outcomes in 90-day and 1-year studies are closer. Table 2 summarizes the data for chemicals for which dog NOAELs were lower in longer studies (that is, the one-year NOAEL is lower by a greater than twofold 


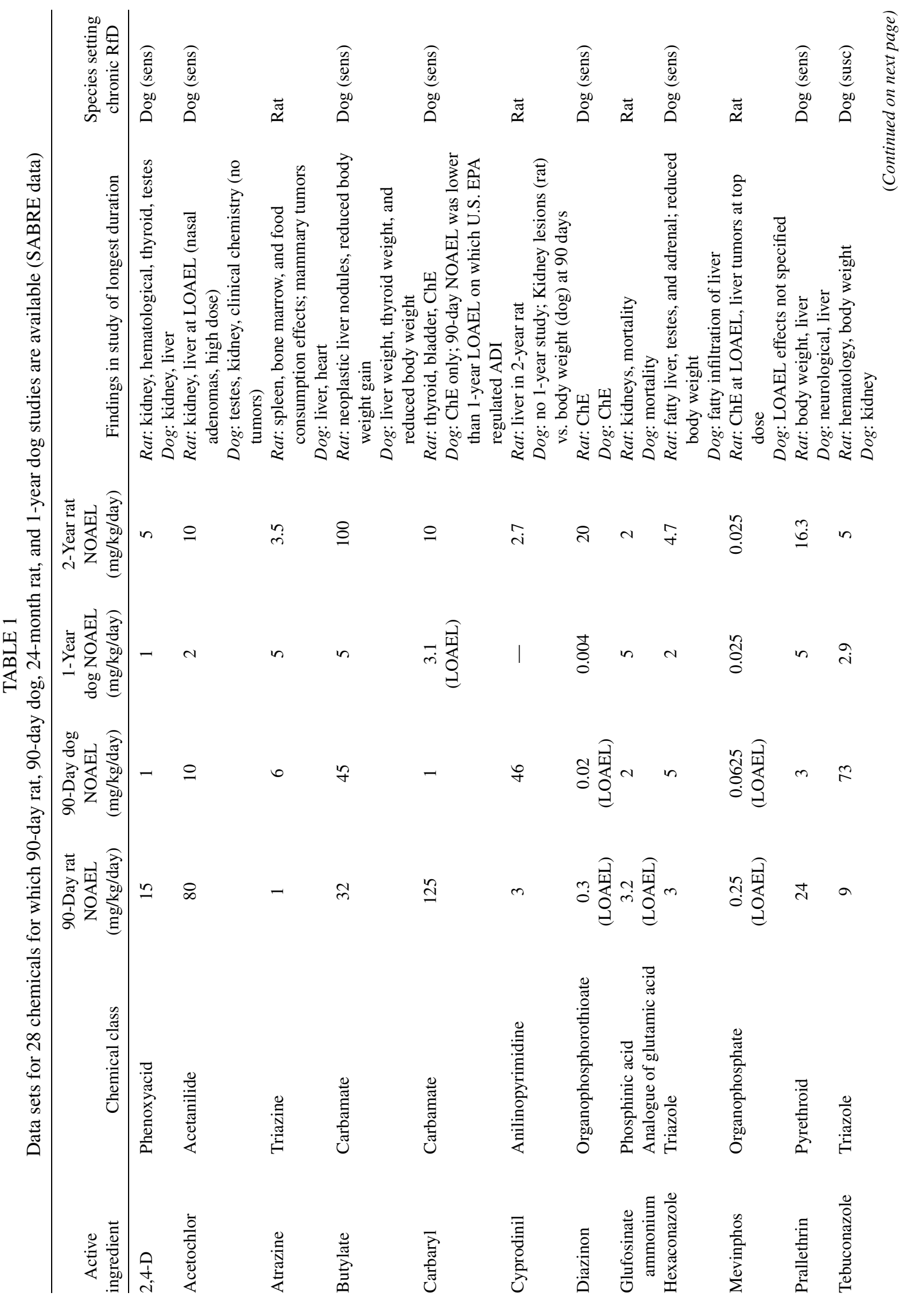




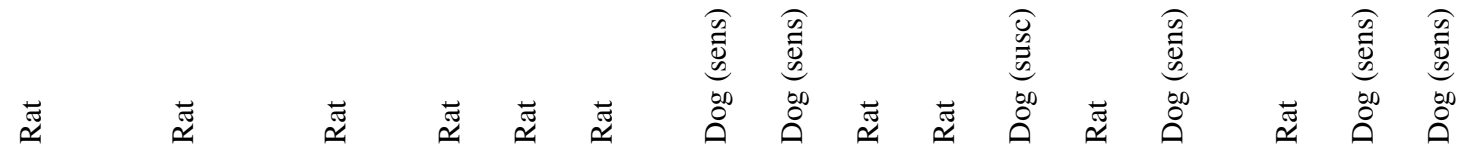

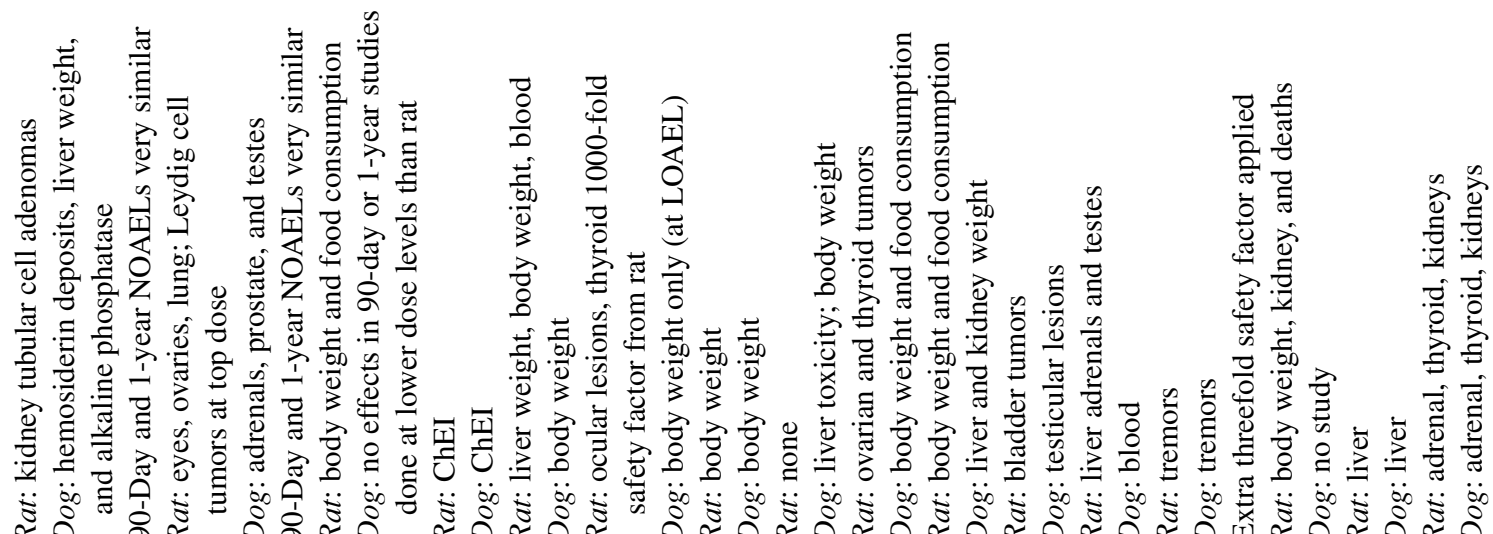

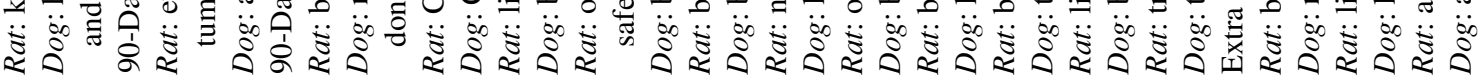

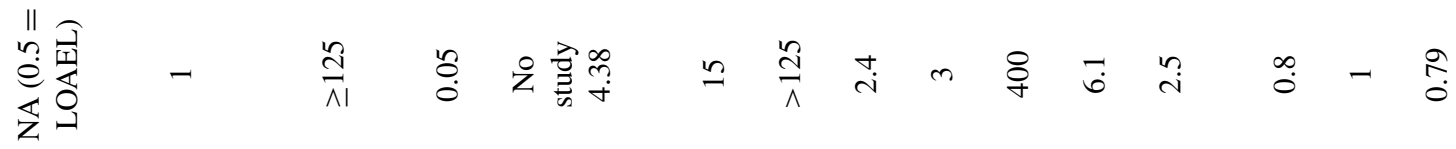

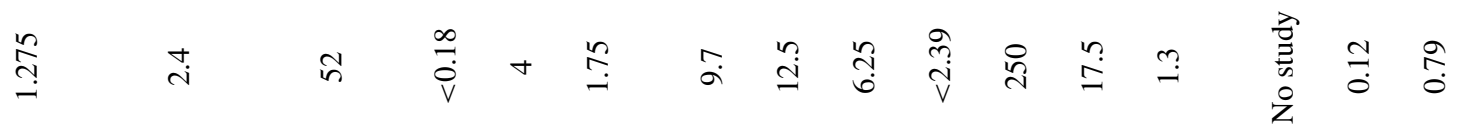

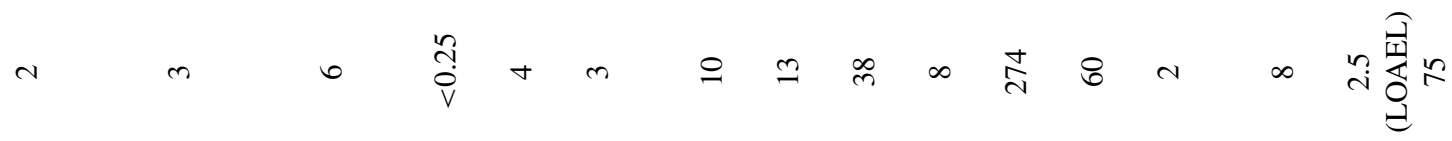

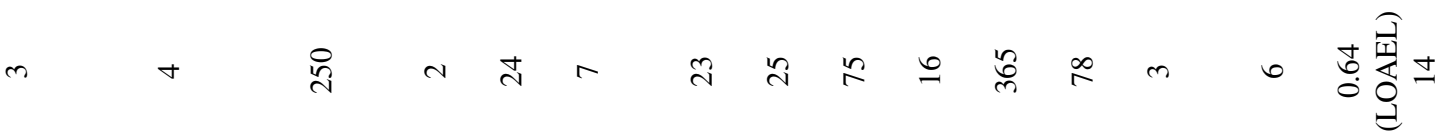

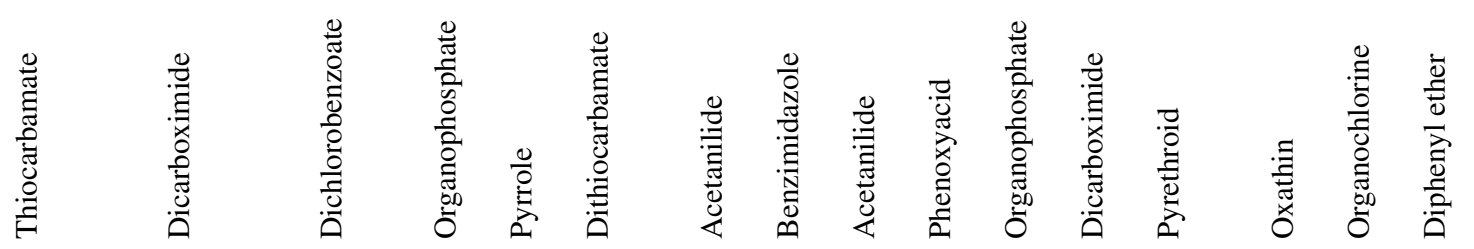

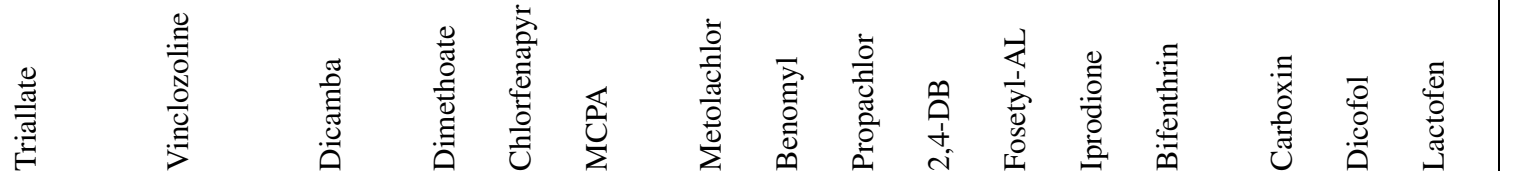


TABLE 2

Chemicals for which dog NOAELs were lower in longer exposure studies (SABRE data)

\begin{tabular}{lccccc}
\hline & $\begin{array}{c}\text { 90-Day dog } \\
\text { NOAEL } \\
\text { Active ingredient } \\
(\mathrm{mg} / \mathrm{kg} / \mathrm{day})\end{array}$ & $\begin{array}{c}\text { 1-Year dog } \\
\text { NOAEL } \\
(\mathrm{mg} / \mathrm{kg} / \mathrm{day})\end{array}$ & $\begin{array}{c}\text { Species used in } \\
\text { setting the ADI }\end{array}$ & $\begin{array}{c}\text { Ratio dog 90-day: } \\
\text { 12-month NOAELs }\end{array}$ & $\begin{array}{c}\text { Ratio rat 90-day: } \\
\text { 24-month NOAELs }\end{array}$ \\
\hline Acetochlor & 10 & 2 & Dog & 5 & 8 \\
Butylate & 45 & 5 & Dog & 9 & 0.3 \\
Hexaconazole & 5 & 2 & Dog & 2.5 & 0.6 \\
Tebuconazole & 73 & 2.9 & Dog & 25 & 3 \\
Lactofen & 75 & 0.79 & Dog & 70 & 5 \\
2,4-DB & 8 & $<2.39$ & Rat & 4 & 12 \\
Iprodione & 60 & 17.5 & Rat & 4 & 30 \\
Propachlor & 38 & 6.25 & Rat & 12 & \\
\hline
\end{tabular}

difference, excluding the three chemicals for which no NOAEL was determined in the 90-day study). The data show that for the 27 chemicals for which there were both 90-day and 1-year dog studies, in only 8 cases were the 1 -year values lower. In three of these cases, the chemicals were regulated on the outcome in the rat as the most sensitive species, making data generated in the dog redundant for the purpose of setting the chronic RfD.

A further analysis of the remaining five chemicals showing lower 1-year dog NOAELs has been undertaken to study the potential influence of dose selection on the outcome of the studies. Table 3 shows LOAELs from the respective 90-day and 1-year studies for these chemicals:

- For hexaconazole, the difference in outcome is within threefold and confirmed by both NOAELs and LOAELs.

- Two 1-year studies have been conducted for acetochlor that give differing outcomes, with one of the studies within twofold of the outcome of the 90-day study.

- The lower 1-year NOAELs for tebuconazole and lactofen cannot be ascribed to dose selection artifacts.

The capability to produce lower NOAELs in studies of longer duration could be a property of the chemical. Rat 90-day and

TABLE 3

Impact of dose selection on dog LOAELs in 90-day and 1-year studies (SABRE data)

\begin{tabular}{lcc}
\hline Active ingredient & $\begin{array}{c}\text { 90-Day dog } \\
\text { LOAEL } \\
(\mathrm{mg} / \mathrm{kg} / \mathrm{day})\end{array}$ & $\begin{array}{c}\text { 1-Year dog } \\
\text { LOAEL } \\
(\mathrm{mg} / \mathrm{kg} / \mathrm{day})\end{array}$ \\
\hline Acetochlor & 60 & $12 / 40$ \\
Butylate & $>45$ & 20 \\
Hexaconazole & 25 & 10 \\
Tebuconazole & 352 & 4.4 \\
Lactofen & 250 & 4 \\
\hline
\end{tabular}

24-month data were compared to determine if this reduction in NOAEL with increasing duration was also seen in the rat (Table 2). Five of the eight chemicals have a similar ratio of NOAELs between the longer and shorter term studies for both species. For hexaconazole, the NOAELs for all four studies are within a range of $2-5 \mathrm{mg} / \mathrm{kg}$ (rat 90 -day: $3 \mathrm{mg} / \mathrm{kg}$; rat $24-m o n t h$ : $4.7 \mathrm{mg} / \mathrm{kg}$; dog 90-day: $5 \mathrm{mg} / \mathrm{kg}$; and dog 12 -month: $2 \mathrm{mg} / \mathrm{kg}$ ), which suggests that the variations may be caused by dose setting. For tebuconazole, the dog 90-day NOAEL is considerably higher than the other three NOAELs (rat 90 -day: $9 \mathrm{mg} / \mathrm{kg}$; rat 24-month: $5 \mathrm{mg} / \mathrm{kg}$; dog 90-day: $73 \mathrm{mg} / \mathrm{kg}$; and dog 12-month: $2.9 \mathrm{mg} / \mathrm{kg}$ ). Butylate is difficult to understand, with the dog $12-$ month NOAEL being considerably lower than the other three NOAELs (rat 90-day: $32 \mathrm{mg} / \mathrm{kg}$; rat 24-month: $100 \mathrm{mg} / \mathrm{kg}$; dog 90-day: $45 \mathrm{mg} / \mathrm{kg}$; and dog 12-month: $5 \mathrm{mg} / \mathrm{kg}$ ), although the LOAEL is $20 \mathrm{mg} / \mathrm{kg}$.

Further analyses of the use of the dog and the optimum study duration are described in this paper (see Refinement of the Proposal: Role of the Second Species).

Studies of Carcinogenicity in the Mouse. In the data set (shown in Table 5), there was one chemical for which 2-year rat and mouse carcinogenicity studies were not available (chlorfenapyr), and there were four chemicals for which there was no mouse carcinogenicity study. The chemicals were classified as shown in Table 4, and they are identified as shown in Table 5.

Tumors were detected in the rat, with 13 of the 27 chemicals remaining in the analysis ( 6 of these also induced tumors in the mouse). An additional four chemicals induced mouse tumors in the absence of findings in the rat; however, all but one of these chemicals resulted in liver tumors. The modes of action for these liver tumors were of questionable or no relevance to humans.

\section{The Relationship between 28- and 90-Day Rat Data}

Apart from a study carried out by the European Crop Protection Association (ECPA, 1999), there are few data on the relationship between the no-observed-effect levels (NOELs) in the rat for study durations of 28 days and 90 days. Twenty-eight-day 
TABLE 4

Presence of tumors in studies in the SABRE database

\begin{tabular}{lcc}
\hline Classification & Identifier & Number (\%) \\
\hline No tumors in rat or mouse & $\mathrm{N}$ & $10(37 \%)$ \\
Liver tumors in mouse only & $\mathrm{ML}$ & $3(17 \%)$ \\
$\begin{array}{l}\text { Tumors other than liver } \\
\text { tumors in mouse only }\end{array}$ & $\mathrm{MO}$ & $1(4 \%)$ \\
$\begin{array}{l}\text { Tumors only in rat } \\
\text { Tumors in both rat and } \\
\text { mouse }\end{array}$ & $\mathrm{R}$ & $7(26 \%)$ \\
\hline
\end{tabular}

studies are commonly performed for dose-setting purposes for 90-day studies, and often do not include a full range of observations. Group sizes may be five rather than 10 . In addition, an expectation that the NOAEL will be lower in the subsequent 90day study will often cause the study director to choose a set of dose levels lower than the dose levels used in the 28-day study. It is common, for instance, to divide all the dose levels used in the 28-day study by a factor of 2 or 3 when moving to the 90-day study. Therefore, it is difficult to relate the results of these studies to their respective 90-day studies. Even so, the ECPA data suggest that a factor of three would be adequate to extrapolate between a 28-day rat and a 90-day rat study (Figure 1).

\section{Summary of Analysis of the SABRE Database}

In summary, analysis of the SABRE database indicates that:

- The rat and the dog can respond with a differential sensitivity-that is, the same effects occur but at different dose levels - and less commonly with a different susceptibility - that is, different effects occur - to the same chemical. Both species should be retained as test species for evaluating the systemic toxicity of agricultural chemicals.

- Longer duration studies (1 year) in the dog do not result in appreciably lower NOAELs or identify new effects for the majority of chemicals when compared to the shorter duration study (90 day) in this species (20 out of 28 cases). This may be because the difference in the proportion of the lifespan in the dog between 90 days and 1 year $(2.5 \%$ and $10 \%$, assuming a 10 -year lifespan) is relatively small when compared to a 90 day rat study and a 24-month rat study (10\% and $80 \%$, assuming a 2.5-year lifespan). However, when there is a decrease in NOAEL between the 90-day and 12-month dog studies, there is a decrease in NOAEL in the rat between the 90-day and 24-month NOAELs in 5 out of 8 cases.

- The additional information provided by the mouse carcinogenicity study is of very limited additional value in risk assessment.

\section{DEVELOPMENT OF A TIERED APPROACH}

\section{Requirements and Challenges for the Development of a Tiered Approach}

Safety assessment of agricultural chemicals has, as its goal, the facilitation of risk assessments for consumers, operators, bystanders, residential uses, and others by identifying potential adverse health effects. These effects must be characterized in terms of variation of dose, duration of exposure, and route of exposure. It should then be possible to derive a human exposure level that should not be exceeded for a particular exposure situation.

The challenge taken on by the Task Force was to improve the ability of the data set to provide relevant information for the variety of risk assessments required, while at the same time reducing the number of animals needed for testing.

The Task Force developed a series of studies that achieves the following:

- The series of studies provides data of direct relevance for a range of less-than-lifetime durations of human exposure that have been defined as being required for risk assessments.

- The series allows for extrapolation from route to route by utilizing ADME and a limited number of studies by the dermal and inhalation routes.

- The series includes studies of short duration and "recovery periods" that can provide information of relevance to intermittent exposures.

- The series covers a range of specific toxic effects that can be assessed as appropriate to provide a more refined evaluation of potential hazards.

The key elements of the series of studies are as follows:

- Studies are of appropriate duration to relate to human exposure durations of relevance to risk assessment. These studies incorporate a full set of toxicological endpoints, whatever their duration. The shorter term studies provide data for appropriate risk assessments in their own right. This contrasts with the current testing program in which shorter term studies are often preliminary to longer term studies, and are designed only to provide dose-setting information.

- Measurements of plasma levels of parent or appropriate metabolites are taken for assessment of internal dose, which aids in species-to-species and route-to-route extrapolation.

- Recovery periods are included to aid with assessment of intermittent exposures.

- Indicators for specific effects that lead to specialized studies are included that will further characterize the response if positive, but that will provide reasonable assurance of the absence of concern if the indicators are not triggered. 
TABLE 5

Tumor findings for 28 chemicals in the SABRE database

\begin{tabular}{|c|c|c|}
\hline Active ingredient & Chemical class & Tumor findings \\
\hline 2,4-D & Phenoxyacid & $\begin{array}{l}\text { No tumors } \\
\mathrm{N}\end{array}$ \\
\hline Acetochlor & Acetanilide & $\begin{array}{l}\text { Nasal papillary adenomas in the rat; liver and lung tumors in the } \\
\text { mouse at } \geq 75 \mathrm{mg} / \mathrm{kg} / \mathrm{day} \\
\mathrm{R}\end{array}$ \\
\hline Atrazine & Triazine & $\begin{array}{l}\text { Mammary tumors in the rat at } 50 \mathrm{mg} / \mathrm{kg} / \mathrm{day} \\
\text { No mouse carcinogenicity } \\
\mathrm{R}\end{array}$ \\
\hline Butylate & Carbamate & $\begin{array}{l}\text { Tumors in rat only } \\
\mathrm{R}\end{array}$ \\
\hline Carbaryl & Carbamate & $\begin{array}{l}\text { Tumors in the rat at } 350 \mathrm{mg} / \mathrm{kg} / \text { day; liver and kidney tumors in the } \\
\text { mouse at } 1249 \mathrm{mg} / \mathrm{kg} / \mathrm{day} \\
\text { RM }\end{array}$ \\
\hline Cyprodinil & Anilinopyrimidine & $\begin{array}{l}\text { No tumors } \\
\mathrm{N}\end{array}$ \\
\hline Diazinon & Organophosphorothioate & $\begin{array}{l}\text { No tumors } \\
\text { No mouse carcinogenicity } \\
\text { N }\end{array}$ \\
\hline Glufosinate ammonium & $\begin{array}{l}\text { Phosphinic acid analogue } \\
\text { of glutamic acid }\end{array}$ & $\begin{array}{l}\text { No tumors } \\
\text { No mouse carcinogenicity } \\
\text { N }\end{array}$ \\
\hline Hexaconazole & Triazole & $\begin{array}{l}\text { Leydig cell tumors in rat at } 47 \mathrm{mg} / \mathrm{kg} / \text { day } \\
\mathrm{R}\end{array}$ \\
\hline Mevinphos & Organophosphate & $\begin{array}{l}\text { Hepatocellular carcinomas in the rat at } 0.6 \mathrm{mg} / \mathrm{kg} / \mathrm{day} \\
\text { No mouse study in database } \\
\mathrm{N}\end{array}$ \\
\hline Prallethrin & Pyrethroid & $\begin{array}{l}\text { No tumors } \\
\mathrm{N}\end{array}$ \\
\hline Tebuconazole & Triazole & $\begin{array}{l}\text { No tumors } \\
\text { Mouse-liver tumors at top dose of } 279 \mathrm{mg} / \mathrm{kg} / \mathrm{day} \\
\text { ML }\end{array}$ \\
\hline Triallate & Thiocarbamate & $\begin{array}{l}\text { Tumors in rat; kidney tubular cell adenomas at } 0.5 \mathrm{mg} / \mathrm{kg} / \mathrm{day} \\
\text { Mouse-hepatocellular carcinomas at } 9 \mathrm{mg} / \mathrm{kg} / \mathrm{day} \\
\text { RM }\end{array}$ \\
\hline Vinclozoline & Dicarboximide & $\begin{array}{l}\text { Leydig-cell tumors in rat at top dose } \\
\text { Liver tumors in mouse at } 1411 \mathrm{mg} / \mathrm{kg} / \text { day } \\
\text { RM }\end{array}$ \\
\hline Dicamba & Dichlorobenzoate & $\begin{array}{l}\text { None } \\
\mathrm{N}\end{array}$ \\
\hline Dimethoate & Organophosphate & $\begin{array}{l}\text { None } \\
\mathrm{N}\end{array}$ \\
\hline Chlorfenapyr & Pyrrole & None-no 2-year rat or mouse carcinogenicity studies in database \\
\hline MCPA & Dithiocarbamate & $\begin{array}{l}\text { Rat thyroid follicular cell tumors at high dose } \\
\mathrm{R}\end{array}$ \\
\hline Metolachlor & Acetanilide & $\begin{array}{l}\text { Rat liver tumors at LOAEL } \\
\mathrm{R}\end{array}$ \\
\hline Benomyl & Benzimidazole & $\begin{array}{l}\text { Mouse liver tumors at LDT }(75 \mathrm{mg} / \mathrm{kg} / \text { day }) \\
\text { ML }\end{array}$ \\
\hline
\end{tabular}


TABLE 5

Tumor findings for 28 chemicals in the SABRE database (Continued)

\begin{tabular}{lll}
\hline Active ingredient & \multicolumn{1}{c}{ Chemical class } & \multicolumn{1}{c}{ Tumor findings } \\
\hline Propachlor & Acetanilide & Rat ovarian and thyroid tumors and mouse liver tumors \\
& Phenoxyacid & RM \\
2,4-DB & Organophosphate & $\mathrm{N}$ \\
Fosetyl-AL & Dicarboximide & Rat bladder tumors \\
& & $\mathrm{R}$ \\
Iprodione & Pyrethroid & Rat testicular tumors; mouse liver tumors \\
& & RM \\
Bifenthrin & Assorted tumors in the mouse \\
& Oxathin & MO \\
Carboxin & No tumors \\
Dicofol & Organochlorine & N \\
Lactofen & Diphenyl ether & $\mathrm{N}$ \\
& & Liver tumors in the mouse \\
\hline
\end{tabular}

Note: See Table 4 for explanation of identifiers (N, ML, MO, R, and RM).

\section{Basic Concept: Matching Duration of Study with Duration of Human Exposure}

The Task Force devised a set of studies which would provide information for the following human exposure periods:

- One day.

- Two to 28 days.

- One to 6 months.

- Greater than 6 months.

Animal Study to Provide Data Relevant to 1-Day Human Exposure. A single-dose study is proposed with full evaluation at 24 hours and 7 days, with histology, clinical chemistry, hematology and other specialized investigations that may be indicated by structure activity or information from other studies.

\section{8-day rat vs 90-day rat}

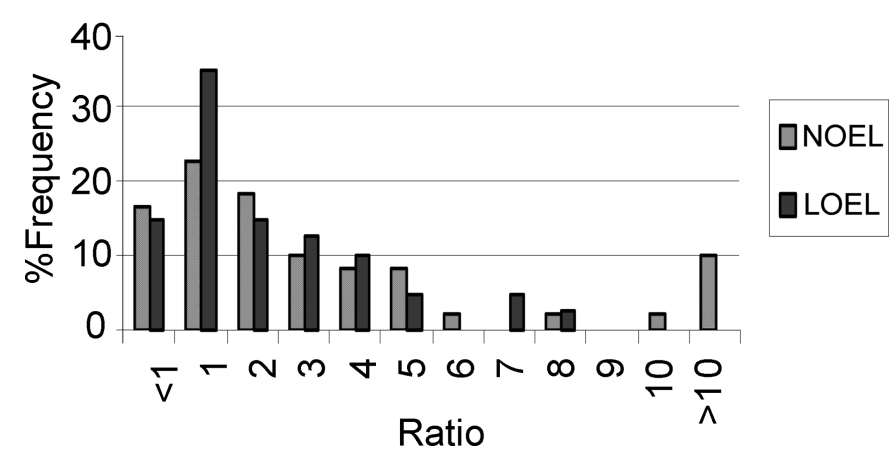

FIG. 1. Ratio of NOELs and LOELs from ECPA data (ECPA, 1999).
Animal Study to Provide Data Relevant to 2 to 28 Days (2 to 7 Days and 1 to 4 Weeks) of Human Exposure. At first evaluation, the Task Force divided this time period of human exposure into 2 to 7 days and 1 to 4 weeks. A 7-day study was thought to be the appropriate study for the 2- to 7-days exposure, and a 28-day study to be appropriate for 1 to 4 weeks. Ultimately, in an effort to minimize animal use, the Task Force recommended the use of one study, based on a common view that the difference in toxicity would not be significant. (A preliminary suggestion was a 14-day study; however, reservations existed. The 14-day study duration does not allow observation of some delayed effects, such as some types of delayed, or peripheral, neuropathy.) A 28-day study was therefore selected to cover the period from 2 to 28 days of human exposure. The study includes histology, clinical chemistry, hematology, and other specialized investigations that may be indicated by structure activity or information from other studies. It would include groups of animals treated for 28 days and then removed from treatment for a further 14 days before being terminated, in addition to those animals terminated immediately after 28 days of treatment. This protocol would allow for the progression or regression of effects to be assessed.

Animal Study to Provide Data Relevant to 1 to 6 Months of Human Exposure. A 90-day study was selected as being the appropriate study for 1 to 6 months of human exposure. The study includes histology, clinical chemistry, hematology, and other specialized investigations that may be indicated by structure activity or information from other studies.

Animal Study to Provide Data Relevant to Greater Than 6 Months of Human Exposure. A 12-month study was selected to be the appropriate study for longer than 6 months of exposure in humans. The study includes histology, clinical chemistry, 
hematology, and other special investigations that may be indicated by structure activity or information from other studies. There is no recovery group because the study duration is intended to cover effects for an extended period of time and will not contribute to an understanding of intermittent exposure.

At this stage of proposal development, the full study package consisted of eight studies: four in the rat and four in the dog. The Task Force proposed that the first study to be performed should be the 28-day rat study, which would provide background data in a reasonable period of time that is relevant for 2 to 28 days of human exposure. The Task Force then considered whether all of these studies would be necessary in all cases. It considered two factors: the role of the second species and the influence of potential human exposure at each of the human exposure durations.

\section{Refinement of the Proposal: Role of the Second Species}

The dog is the second species that is used most frequently in agricultural chemical safety assessment. It is a regulatory requirement, except in special circumstances in which it can be demonstrated that the dog is not relevant to humans. The second species covers the possibility that the rat may be insensitive to an effect of a compound to which humans are more sensitive. The use of a second species that is phylogenetically removed from the rat increases the likelihood that one or the other of the species will be at least as sensitive as humans.

The SABRE database allowed the Task Force to determine how often the dog was more sensitive than the rat (i.e., the NOAELs and LOAELs derived in the dog were lower than in the rat) and/or of different susceptibility (i.e., different effects were observed). The conservative and health protective assumption is that the dog is the more relevant model in these cases for extrapolation to humans, and the derivation of RfDs is, therefore, from the dog data. In cases in which the dog is of different susceptibility, the effects should be considered when selecting the uncertainty or safety factor to be applied.

Figures 2 and 3 show the results of the interrogation of the SABRE database comparing the NOAELs and LOAELs derived from rat and dog studies with the same compounds. In each figure, a ratio of less than 1 indicates that the rat was more sensitive, and a ratio of more than 1 indicates that the dog was more sensitive. Inspection of the figures reveals that at 90 days there is

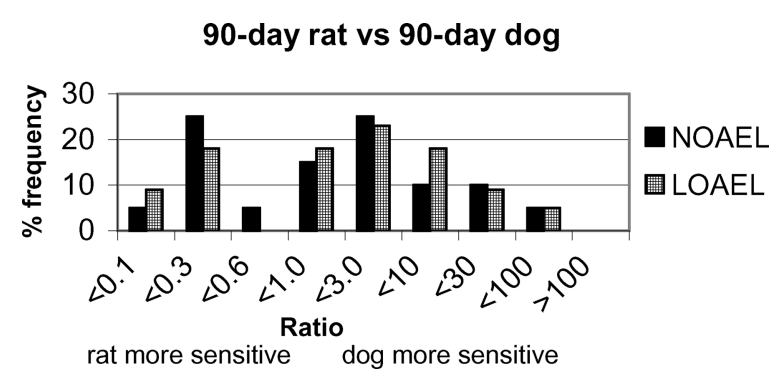

FIG. 2. Comparison of NOAELs and LOAELs in 90-day rat and 90-day dog studies.

\section{2-year rat vs 12-month dog}

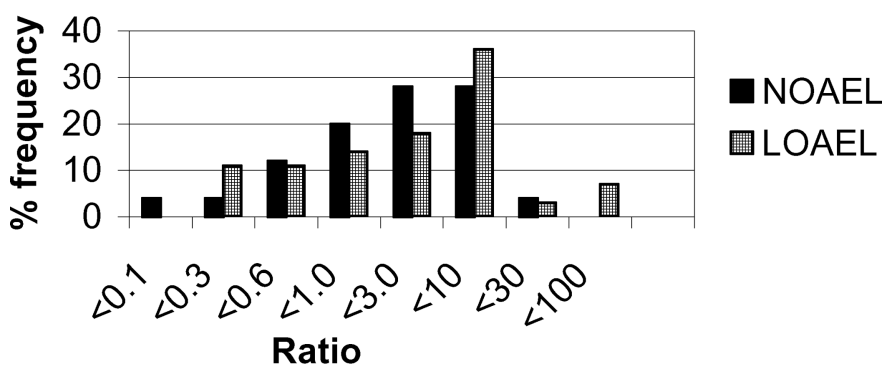

rat more sensitive dog more sensitive

FIG. 3. Comparison of NOAELs and LOAELs in 24-month rat and 12-month dog studies.

an approximately normal distribution of sensitivity around unity $(5 / 19$, i.e., $26 \%$ cases have ratios between 0.6 and 2), with an equal number of situations ( $7 / 19$, i.e., $37 \%$ cases) in which the dog is more sensitive than the rat (ratios above 2), and the rat is more sensitive than the dog (ratios below 0.6). Therefore, the dog would be the species from which the RfD for 1 to 6 months of exposure would be derived approximately one-third of the time. The distribution for the longer term comparison is of a different shape, but there is still a significant number of compounds for which the dog is more sensitive. On the basis of this evidence, the Task Force concluded that the dog could not be excluded as a second species.

Once the need for the dog as a second species had been established, thought was then given to how best to use the dog in the new approach to safety assessment. Advantages to using the dog include its utility as a better model for cardiovascular effects and the ability to make assessments several times in the same animals during the course of a study. It is also possible to take serial blood samples from dogs to better understand toxicokinetics. The purpose of evaluation in the dog can be summarized as follows:

- To determine whether there are significant new effects in the dog when compared to the rat.

- To determine whether the dog displays the same effects but is more sensitive.

- To assess effects on the cardiovascular system and possibly other systems as necessary.

- To assess toxicokinetics, including the option of assessing kinetics after dermal exposure during preliminary studies leading to a main study.

The Task Force agreed that performing a 90-day study in the dog meets the objective concerning the relative sensitivity of the rat and the dog, while at the same time providing information that would be relevant for the 1 to 6 months human exposure period. The study outline would include: 
- 90-Day capsule or dietary dosing.

- Toxicokinetics - six time points on day 1 , week 4 , and week 13.

- Blood pressure and ECG-prestudy, day 1, week 4, and week 13.

- Clinical chemistry and hematology_prestudy, day 1 , week 4, and week 13.

- Pathology.

The 90-day dog study should be a core study and the second study carried out after the 28-day rat study.

If concordance exists between the 28-day rat and the 90-day dog studies in terms of effects seen and the dose levels at which the effects occurred, then either species could be used to further define the toxicity of the compound. In this case, the default is to use the rat for further studies. In reaching this conclusion, it is advisable to consider the "internal dose" for each species derived from toxicokinetics, rather than the "external" or applied dose. In addition, it should be borne in mind that the dog study is of a longer duration. It would be expected that the NOAEL in the longer study could be lower, especially for compounds with a long half-life or for compounds causing effects that are the result of cumulative insult or injury. These factors are important in determining whether the dog is the more sensitive species.

If the rat displayed effects at lower doses than the dog, then the rat should be used in further evaluation of the compound. If the dog showed different effects at lower doses or the same effects at lower doses, then the dog should be considered for further evaluation.

\section{Assessing the Effects of Short-Term Human Exposure}

The assessment of a single-day human exposure should be done after the results of the 28-day rat study and the 90-day dog study are available. These studies should indicate whether the dog or the rat should be used for the assessment as described earlier, taking into account the relevance of the endpoints for acute exposure. It is also appropriate to consider the projected human exposure at this stage. One would expect that toxicity in terms of effects and dose levels observed after 1-day exposure would be less than after 28- or 90-day exposure at the same dose level. If the RfD derived from the 28-day rat and 90-day dog study indicates an adequate margin of exposure, then the requirement to perform a single-dose study could be waived. However, the decision to perform a single-dose study often needs to be made early in the development of an agricultural chemicalthat is, before the chemical's use rate and all of its potential outlets have been determined. This may limit the opportunity to waive the single-dose study on exposure grounds. However, if the key effects observed in the dog can be assessed by the parameters that will be measured on day one of the study (ECG, blood pressure, clinical chemistry, clinical observations), then the requirement to perform a single-dose study may be waived. In addition, the single-dose study will be valuable in providing insight on the kinetics (speed of onset, recovery period) of the effect caused by the compound. This information can be used to assess intermittent and variable exposure as described later (see The Proposed Tiered Testing Approach, Step 7). For these reasons, it is expected that a single-dose study will be performed more often than not. If such a study is done, then it should conform to the design just outlined. It should never be necessary to perform both the rat and the dog single-dose study.

\section{Assessing the Effects of Longer Term Human Exposure}

The first step in assessing longer term (i.e., over 6 months) human exposure is to determine whether the use pattern of the compound will give rise to exposure longer than 6 months. Such a scenario is likely to be the case for compounds that are used throughout the year in tropical conditions, or for compounds with residues in food or drinking water. If longer term exposure is not predicted, then the requirement for longer term studies may be waived. Exposures to most agricultural chemicals will be longer term; however, there may be exceptions, such as rodenticides.

The Task Force determined the appropriate study duration for chronic exposure to be 12 months, which represents an appreciable fraction of the life span of the rat. Initially, the Task Force was of the view that the results of the 28-day rat and 90day dog studies should be taken into account in deciding which species to use for 12-month chronic toxicity studies. If the rat was considered to be the more sensitive species, then the rat should be used and, conversely, if the dog were the more sensitive species, then the dog should be used. However, there is a growing body of evidence to show that extending the duration of exposure in dogs from 3 months to 12 months provides no useful additional data (Spielmann and Gerbracht, 2001). In addition, 12 months is only a small fraction of the life span of the dog. Interrogation of the SABRE database supports these conclusions. There were very few (two) cases in which differences in sensitivity could not be attributed to the choice of lower dose levels in the 12-month study. Dose levels in 12-month studies are generally set slightly lower than in the preceding 90-day studies on the assumption that effects will progress during the longer duration; however, this assumption is not borne out in practice. Nor were there any differences in the nature of the effects observed. The Task Force recommends the use of the 12-month rat study, carried out as an interim sacrifice in a 24-month carcinogenicity study (see later discussion). Twelve months was chosen rather than 24 months for this assessment because by 2 years, age-related changes make interpretation of the data difficult, and any noncancer effects should be evident after 12 months. However, animals will be monitored for up to 2 years for any noncancer effects that emerge after 1 year. The RfD based on systemic toxicity for exposures of greater than 6 months should be set using the lower of the NOAELs from the 12-month rat chronic/24-month rat bioassay or the 90-day dog study.

The Task Force had concerns about the number of dogs used in 90-day studies. The current minimum recommended 
by the Organisation for Economic Cooperation and Development (OECD) in its Test Guideline Number 409 (OECD, 1998) is four per sex per group, which can cause problems of interpretation and loss of sensitivity if even one dog is withdrawn from the study due to ill health or if one dog shows outlying values. Taking into account statistical power, logistical issues in animal husbandry, and humane considerations, the Task Force recommends increasing the group size to six per sex per group in the 90-day study.

\section{The Relevance of Experimental Animal Study Duration to Human Exposure Duration}

There are a number of factors that need to be taken into account when considering the relevance of the duration of an experimental animal study to the duration of human exposure to the same chemical. The simplest approach is to directly extrapolate the exposure duration in animals to humans; that is, a 1-day exposure in an animal would be equivalent to 1 day in a human, 28 days in animals would be equivalent to 28 days in humans, and so on. This appears to be satisfactory until the timescale is extended, especially if the question is asked a different way; that is, "What is the correct animal exposure duration to model several years of exposure in humans?" The situation then becomes complicated by factors such as aging and percentage of life span.

If there were no difference between durations of exposure in animals and humans, then it would not be necessary to consider performing studies of different durations. Clearly, this is not the case. In order to seek better insight into this issue, the factors that might change the nature of the response when a chemical is given for different lengths of time need to be examined.

Three major factors should be taken into account:

- The toxicokinetics of the chemical.

- The toxicodynamics of the response elicited by the chemical.

- The influence of the proportion of the life span of the subject on these factors.

The interaction of toxicokinetics (what the organism does to the chemical) and toxicodynamics (what the chemical does to the organism) has been discussed by Rozman and Doull (2000) in terms of the time element of toxicity. They define toxicity as "the accumulation of injury over short or long periods of time, which renders the organism incapable of functioning within the limits of adaptation." The authors suggest that Paracelsus' famous quote should be supplemented to read "Dose and time together make the poison." Rozman and Doull identified the factors that underlie toxicokinetics and toxicodynamics:

Toxicokinetics:

- Absorption.

- Elimination.
- Distribution.

- Biotransformation,

- Excretion.

Toxicodynamics:

- Injury.

- Recovery.

- Adaptation.

a Repair.

- Reversibility.

Rozman and Doull point out that although the basic relationship is concentration $\times$ time $=k$, with this number of variables available for interaction, the responses will be varied and complicated to model. However, the extremes of variability can be predicted; that is, no elimination will lead to linear accumulation of compound; no recovery will lead to linear accumulation of injury. Each chemical will have its own unique characteristics, depending on how the variables interact. If all of the variables were known, it would be possible to predict the toxicity of a compound without further experimentation.

Both toxicokinetics and toxicodynamics have their own internal time dependencies that dictate how dose and time will interact. These time dependencies can vary from minutes to years depending on the factors involved, but once they are understood, they allow predictions to be made with confidence about the effect of any exposure duration.

The essence of the ACSA Systemic Toxicity approach is an attempt to provide the optimum data base from which the effect of dosing chemicals for different durations of time can be predicted. The time dependencies of the toxicokinetics will be defined as described by the ACSA ADME Task Force (Barton et al., 2006). The studies of different durations with recovery define the range of toxic effects and the time needed to cause them or for them to be manifest. It will therefore be possible to deduce the time dependencies of the toxicodynamics from knowledge of the overall toxicity and the toxicokinetics.

The question then remains; "How should the time dependencies observed in experimental animals be extrapolated to humans?" There are essentially two ways:

- Unadjusted duration.

- Percentage of life span.

The answer will depend on the effect being considered, but a rule of thumb can be used that states that the shorter the time dependency of an effect, the more likely it is that unadjusted duration should be used, and the longer the time dependency, the more the percentage of life span comes into play. Carcinogenicity is the effect that is most often considered using a percentage of lifetime approach. It could, however, be argued that the most appropriate factor is not the percentage of life span, but the influence of life stage. The toxicodynamics of cancer 


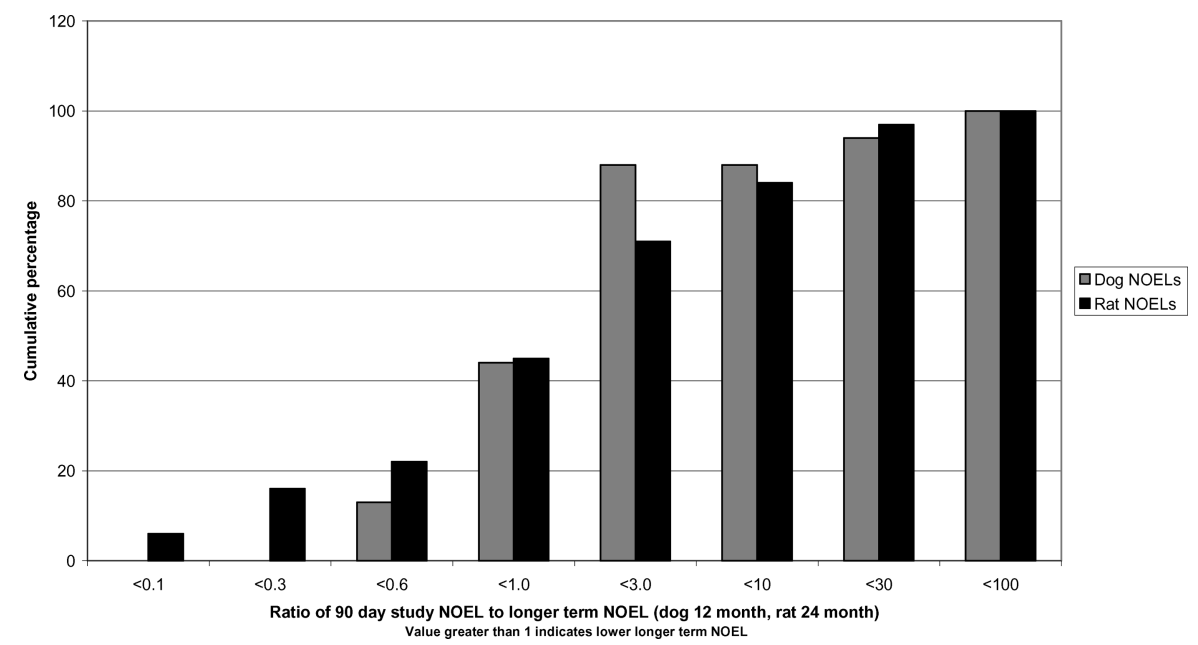

FIG. 4. Comparison of ratios for 90-day studies in rats and dogs to longer term studies in the same species.

could be oversimplified to be described as an initial injury that leads to cancer later in life when the repair mechanism, which is stopping progression, breaks down. The most potent carcinogens can cause early tumors or can cause tumors later in life following a brief exposure early in life because of the magnitude of the injury (and the mechanism/mode of action). In the experimental models for carcinogenicity, an important factor seems to be to impair the repair mechanism either by keeping the animals in the standard rodent bioassay until they reach an age when this occurs "normally" through senescence or by genetically modifying the animals as in the shorter term mouse models.

Other effects that take a long time to occur, such as the induction of lung fibrosis, which could be characterized as a misdirected repair attempt, seem to take the same amount of time to observe whether it is in the dog or the rat, independent of the percentage of the life span of the animal.

If percentage of life span is the dominant factor, then it would be expected that there would overall be a greater decrease in NOELs in rats when comparing 90 -day studies (10\% of a $30-$ month life span) to 24-month studies ( $80 \%$ of life span) than in dogs, where a 90 -day study represents $1.66 \%$ of a 15 -year life span and a 12-month study represents $7 \%$. The limited data set provided by SABRE does not bear this out; there is no difference in the distribution of the ratios of 90-day study to longer term study NOELs between rats and dogs (Figure 4). There could be two reasons for this: that direct extrapolation is the assumption to be made, or that the time dependencies of the toxicodynamics of most effects are such that a steady state is reached after 12 months. There will be some exceptions, such as have been discussed for carcinogenicity, when the life stage changes one of the factors underlying the effect, such as repair capacity.

The range of studies included in the ACSA project has been designed to cover a range of exposure durations. It also covers studies of sufficient length to allow for the development of effects with toxicodynamics with long time dependencies.
The inclusion of the 24-month rat study also allows for the effect of age on kinetic and dynamic factors to be taken into account.

\section{Rationale for Dose Selection}

Dose selection has a profound effect on the results of toxicology studies. A major concern has been the setting of the top dose for a study. The interpretation of studies designed to assess specific toxic effects, such as carcinogenicity or endocrine modulation, is difficult when the specific effect only occurs in the presence of marked systemic or general toxicity. There are two approaches to setting the top dose for such studies: the maximum tolerated dose and the limit dose.

\section{Maximum Tolerated Dose}

History and Definition. By the 1950s, it was generally accepted that small rodents were suitable surrogates for studying the potential effects of chemical exposure in humans. It was not until the 1960s that the concept of the maximum tolerated dose (MTD) became established in the dose selection process for carcinogenesis studies (McConnell, 1995). The major architects of the design and choice of the high dose-or what is now known as the MTD-were Drs. John and Elizabeth Weisberger and Umberto Saffiotti of the National Cancer Institute (NCI). While testing several known human carcinogens, these scientists found that it was necessary to use high dose levels to produce cancer in rodents (McConnell, 1989). They defined the MTD as "the highest dose of the test agent during the chronic study that can be predicted not to alter the animals' longevity from effects other than carcinogenicity" (Sontag et al., 1976). They further stated that the choice of the MTD should be based on the results of a 90-day study where the highest dose caused "no more than a $10 \%$ weight decrement, as compared to the appropriate control group; and does not produce mortality, clinical signs of toxicity, or pathologic lesions (other than those that may be related to a neoplastic response) that would shorten the animal's life 
span.” In 1978, the NCI Carcinogenesis Bioassay Program was transferred to the National Toxicology Program (NTP), where the concept of the MTD further evolved. In 1984, the NTP convened an ad hoc group of scientists experienced in the field of carcinogenesis and toxicology. They were concerned that the Sontag et al. (1976) definition was inadequate, and suggested that it be supplemented with the following: "The MTD should not cause morphologic evidence of toxicity of a severity that would interfere with the interpretation of the study" (NTP, 1984). Unfortunately, while there has been a great deal of knowledge gained since 1984 with regard to how chemicals interact with the body, the definition of the MTD has not since been updated.

Basis for Using the MTD in Carcinogenesis Bioassays. The primary reason, objective, and/or justification for using the MTD in carcinogenesis bioassays is to maximize the likelihood of detecting a rodent carcinogen (National Research Council, 1993). Using the MTD adjusts for the insensitivity of the animal model being used, both biologically and numerically. The typical carcinogenesis bioassay uses only 50 animals per dose group as a surrogate for thousands or even millions of people- the logic being to maximize the dose (within reasonable limits) to the extent possible to make up for the small number of animals. Also, the MTD can help in identifying potentially "weak" carcinogens, particularly those that cause an increased incidence of a neoplasm that has a relatively high background incidence. An additional advantage of using the MTD as the highest dose in a carcinogenesis bioassay is to provide some degree of consistency of design so that one can compare the results of one study to another; that is, the MTD serves as a "benchmark." For example, if the dose (the MTD) showing carcinogenic activity for chemical A is in the range of grams per kilogram compared to a low milligrams per kilogram or even micrograms per kilogram dose for chemical B, one would assume that chemical B was the more "potent" and probably a greater hazard than chemical A, unless there is a clear explanation for the dose difference, such as lack of absorption.

A final advantage of using the MTD as the high dose in carcinogenicity studies is because it is consistent with dosing regimens firmly entrenched in toxicology. For example, when one is interested in the potential reproductive toxicity of a chemical of unknown hazard, one normally uses a dose just below that considered maternally toxic. The same is true when one is interested in the teratologic, immunologic, neurologic, etc. potential of a chemical. Even in vitro studies used to study the genotoxicity of a chemical normally use doses just below the lethal dose, which arguably is equivalent to an MTD. Therefore, the use of an MTD in carcinogenesis bioassays is consistent with the basic tenets of toxicology.

However, the MTD also has some inherent disadvantages. For example, the term "maximum" automatically connotes that an "excess" amount of the chemical is used to produce a given effect. In this way, the MTD can be misleading to individuals who do not have hands-on experience in the study of chemicals. This is particularly true in the eyes of the general public who receive their information through the general media (newspapers and television), which rarely places the MTD in the context of human exposure. One approach to this misconception is to substitute another term for the MTD, such as "minimally toxic dose," but use the same definition (Huff et al., 1994). This approach, however, tends to obfuscate the problem rather than solve it. Another disadvantage is the problem of inconsistent definition. For example, two different groups of investigators viewing the same data can differ in their views regarding whether the MTD was attained.

A further important disadvantage is that it is difficult to interpret the relevance to humans for effects that are found at doses that exceed the MTD. For example, if carcinogenic activity is only observed at doses that clearly exceed the MTD, should that particular chemical be considered an animal carcinogen? Some investigators would say "yes," and others would say "yes, but only at a dose which exceeds the MTD," while others would opt for placing the chemical in a noncarcinogenic category. This is a particular problem for regulators and agencies that have to classify chemicals/materials and place them on "lists." When the compounds in question are carcinogenic by a genotoxic mode of action, there is at least some logic to such thinking. However, when the compounds are not genotoxic, as is now the case with those agricultural chemicals that progress to development, there is no logic in classifying chemicals simply on a carcinogenic response at very high doses.

A final disadvantage is that it may be practically impossible to attain an MTD for chemicals with low toxicity. To compensate for this scenario, practical limits (referred to as the "upper limit") have been accepted for studies using some routes of exposure, for example, $1 \%$ of the diet, extent of solubility in water, or limit of acceptance (palatability). There have also been upper exposure bounds for inhalation studies that, for example, take into account a chemical's explosive potential or a practical maximum for a dust.

Basically, dose influences mechanism and, over a wide range of doses, mechanism can change with changing dose (Counts and Goodman, 1995). Thus, a carcinogenic effect observed at a high dose is not necessarily expected to occur at lower doses, especially when dealing with nongenotoxic chemicals (McClain, 1994).

Proposed Definition of the MTD. The concept of using the MTD as the "highest" dose is still valid, but the definition needs to reflect state-of-the-art science. Effects on body weight, morbidity, mortality, or pathology, when present, will continue to be valid endpoints when evaluating the results of short-term studies for selecting the MTD for longer term studies. However, a more science-based rationale is required.

In a practical sense, the chief concern with the MTD is how the resulting data are used in the risk assessment process (McConnell, 1989). The goal for risk assessment is to extrapolate rodent carcinogenicity data to estimate possible human risks that might occur at much lower levels and frequency of 
exposure. A major problem arises when the accomplishment of this goal is linked automatically to a procedure involving a linear dose-response, no-threshold assumption. Therefore, both the criteria for selection of the high dose used and the no-threshold default criterion must be reevaluated in a critical manner (NTP, 1992). In essence, the primary concern is that testing at the MTD can result in toxicity, perhaps secondary to overload of metabolic pathways, which can result in a carcinogenic effect that would not occur at lower doses. A variety of examples, including genotoxic as well as nongenotoxic compounds, illustrates this concept (reviewed in Counts and Goodman, 1995). Gaylor (2005) analyzed the results of over 500 NTP bioassays. This analysis suggested that almost all of the chemicals selected would produce a statistically significant increase in tumor incidence at the MTD with larger sample sizes. Gaylor concluded that the MTD bioassay screen is not distinguishing between true carcinogens and noncarcinogens. Rather, the current bioassay is possibly serving as a screen for the more potent cytotoxins at the MTD, and not as a screen specifically for carcinogenicity.

The definition of the MTD should also incorporate the findings of properly conducted absorption, distribution, metabolism, and excretion (ADME) studies. For example, if a chemical is poorly absorbed, then an upper limit approach to selecting the MTD would be used. Or if, at a given dose, metabolic saturation is achieved, then that dose would be defined as the MTD.

These concepts are in close agreement with guidance in the U.S. EPA Draft Guidelines for Carcinogen Risk Assessment (U.S. EPA, 2003a): "Other signs of treatment-related toxicity associated with an excessive high dose may include (a) significant reduction in body weight gain (e.g., greater than 10\%), (b) significant increases in abnormal behavioral and clinical signs, (c) significant changes in hematology or clinical chemistry, (d) saturation of absorption and detoxification mechanisms, or (e) marked changes in organ weight, morphology, and histopathology." The Health Effects Division of the U.S. EPA Office of Pesticide Programs also provides greater detail in an interim guidance document (U.S. EPA, 2003b).

\section{The Derivation of Limit Doses}

A limit dose of $1000 \mathrm{mg} / \mathrm{kg} / \mathrm{day}$ is used for most repeatdose studies. The origin of this value is not apparent, but it was probably chosen as a reasonably high dose, and it is lower than single-dose limits of $2 \mathrm{~g} / \mathrm{kg}$ or $5 \mathrm{~g} / \mathrm{kg}$. However, the reduction of the recommended limit dose as study duration increases stops at 28 days. In practice, the limit dose of 1000 $\mathrm{mg} / \mathrm{kg} /$ day results in a very high metabolic load on laboratory animals, and it can lead to liver enlargement and kidney toxicity with otherwise relatively nontoxic chemicals. In turn, this can lead to problems in assessing the results of specific toxicity studies.
The Task Force considered whether it would be possible to set limit doses based on an assessment of the maximum theoretical human exposure level, followed by application of a margin of exposure of 1000 from this level to determine the limit dose for the study durations recommended in this proposal. However, no firm conclusions could be drawn.

\section{Assessing Toxicity That May Not Be Covered in the Basic Studies}

The major aim of the suite of basic studies outlined in Figure 5 is to answer the general question, "What is the range of target organs affected and types of toxicity caused by the compound?" A general screen of clinical conditions and observations, clinical chemistry, hematology, and histopathology of major organs is used to explore this question. However, it should be noted that there are several manifestations of toxicity that may not be covered by these basic studies:

- Local toxicity of the concentrated compound.

- Neurotoxicity.

- Immunotoxicity.

- Endocrine modulation.

- Genetic toxicity.

- Carcinogenicity.

Whenever possible, these specific manifestations of toxicity should be identified by indicators in the basic studies that give a high level of confidence that there is or is not a concern. If there is a concern identified by an indicator in a basic study, then secondtier studies can be performed to further characterize the effect in terms of mode of action. To the extent possible, this philosophy has been incorporated into the basic designs proposed here. The discussion that follows reviews how each of these special areas of toxicity can be addressed.

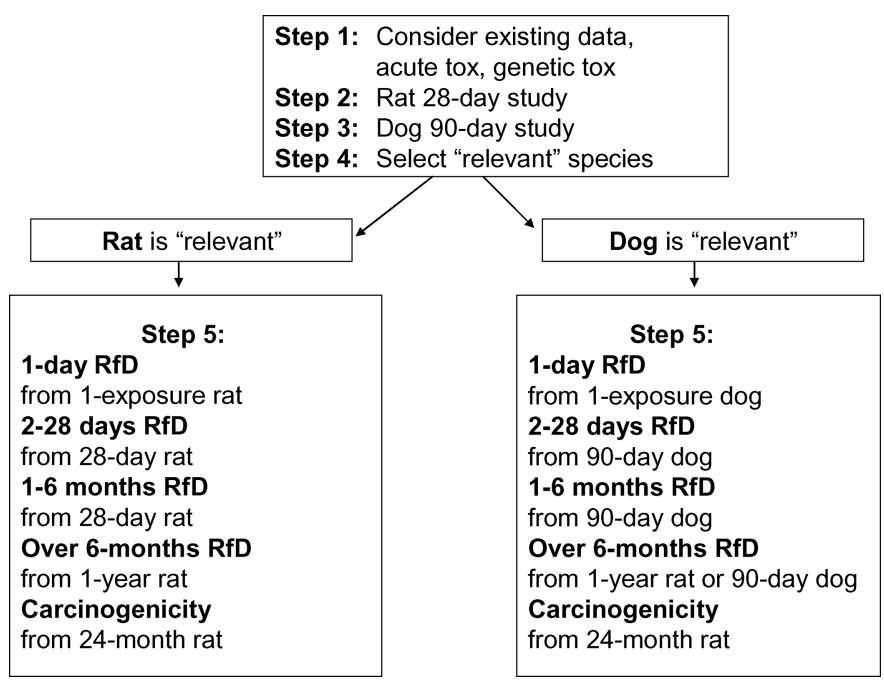

FIG. 5. Diagrammatic representation of the ACSA systemic toxicity tiered strategy. 


\section{Local Toxicity of the Concentrated Compound}

It is important to evaluate the potential effect of the concentrated compound on those who may handle it. If an assessment of oral lethality is necessary for classification purposes, then one of the methods developed by the OECD that assesses lethality while minimizing the use of animals should be employed. The assessment of dermal and ocular irritancy has also been the subject of much recent scrutiny by the OECD. As new methods are introduced in these areas, they should be adopted. Sensitization assays have also been the subject of considerable review. The Local Lymph Node Assay is now the method of choice for active ingredients. However, it should be noted that this method may not be appropriate for assessing the sensitization potential of agricultural chemical formulations because the formulation may run off the mouse ear, giving an underestimate of potency, or the dosing vehicle may break down the formulation and overestimate the sensitizing potential.

The Task Force recommends that OECD methods be used to assess the acute and local toxicity of the concentrated compound.

\section{Neurotoxicity}

The proposed standard 28-day rat and 90-day dog toxicity studies (28/90-day studies) will evaluate functional, behavioral, and morphological endpoints for the nervous system, which may give preliminary or even definitive indications of the neurotoxicity of test materials. Moreover, if the test substance has a neurotoxic mechanism of action, biochemical tests may be performed on the nervous system to assess changes. For example, if the test substance is a cholinesterase inhibitor, neurotoxicity may be assessed in the 28/90-day study by performing cholinesterase measurements in nervous system tissue. Data on the metabolism and pharmacokinetics of the compound will be developed in support of the 28/90-day toxicity study, and will aid in study design, dose selection, and interpretation of the outcome. It is perhaps worth noting that while the halflife of disposition of the compound may be short, the halflife for recovery may be longer. Consequently, it is not possible to assume on the basis of ADME studies that, for example, a 28-day study is not appropriate. Such information will be invaluable in interpreting the result of studies of different duration.

The 28-day rat toxicity study protocol includes both clinical observations (i.e., cage-side monitoring of animals, as well as during handling at the time of dosing or body weight determination), a functional observational battery, and motor activity assessment (Moser and MacPhail, 1990). These behavioral assessments should indicate changes in motor function (e.g., disturbances of gait, abnormal posture, or muscle tone), level of arousal (e.g., hyperactivity, lethargy), autonomic functions (salivation, lacrimation, urination, defecation), psychological status (indicated by stereotypy, aggression, biting, licking, self-mutilation), or pharmacological effects (sedation, anesthesia).
In addition to the extensive functional assessment already described, the 28-day rat study will also include a satellite group of animals for morphological evaluation of the central and peripheral nervous systems following fixation by perfusion.

The neurotoxic assessments included in the 28-day rat and the 90-day dog studies should be sufficient to detect any potential effects on the nervous system. If one or more of the predictive markers is altered by treatment, then more specific tests may be conducted to characterize the toxicity and/or assess potency. These second-tier tests may include, but are not limited to, tests of learning and memory, neurophysiology, or biochemical assessment.

\section{Immunotoxicity}

Assessment of potential adverse effects on the immune system is an important component of the overall toxicity evaluation of chemicals. When appropriate endpoints are included in the study design, evidence of immunotoxicity can be observed in standard toxicology studies such as the proposed core 28-day rat and 90-day dog studies.

Indications of immunosuppression, if expressed, will be evaluated in the proposed core studies as follows:

- Evidence of myelosuppression.

- Alterations in immune system organ weights and histology (e.g., hypocellularity of immune system tissues such as the thymus, spleen, lymph nodes, or bone marrow).

- Decreased serum globulin levels.

- Changes in hematology.

Thus, the following standard clinical and anatomic pathology markers will be included in the 28-day rat study and 90-day dog study specifications: serum biochemical markers such as globulin levels, hematology (including differential), gross pathology findings, immune system-related organ weights, and histologic examination of immune system-related tissues (Basketter et al., 1995).

Histology determinations will include examination of spleen, thymus, lymph nodes, and bone marrow. In addition, the lymphoid tissue that drains or contacts the site of chemical administration (and therefore is likely to be exposed to the highest concentration of the material) should be specifically examined (Basketter et al., 1995). These sites include the gutassociated lymphoid tissues (GALT) for orally administered materials, bronchus-associated lymphoid tissues (BALT), and nasal-associated lymphoid tissues (NALT) for materials administered by the inhalation route, and the regional draining lymph nodes for chemicals administered by the dermal route.

Typically, the outcome of the core studies, including assessment of the endpoints just covered, is sufficient to detect any potential effects on the immune system. If one or more of the 
predictive markers are altered by treatment and this cannot be ascribed as secondary to general systemic toxicity, then consideration should be given to conducting a subsequent functional test (see later discussion). Such a functional test would explore integrated function and further characterize dose response. As with any other form of adverse finding in the core studies, the lack of a NOAEL will require further characterization at lower dose levels in a subsequent study.

Immune Function Studies. When warranted by observations in the core studies, additional studies to determine potential for effects on immune function should be considered. Other considerations are also important to determine whether immune function studies should be conducted to explore the potential adverse effects on immune function. Such considerations include known class effects/SAR and observed pharmacokinetic effects (e.g., high concentrations of chemical and/or metabolites in immune system tissues).

When immune function studies are conducted, the most widely accepted general method is experimental determination of effect on immune response to a T-cell-dependent immunogen (T-cell-dependent antibody response). The antisheep red blood cell (SRBC) primary (IgM) antibody response assay (usually referred to as the "plaque assay") was extensively evaluated by the National Toxicology Program (NTP), and was found to be useful in identifying immunosuppressant chemicals (Luster et al., 1988, 1992a, 1992b, 1993). Modifications of the plaque assay are available that can be used to determine effects on both IgM and secondary $(\mathrm{IgG})$ immune responses to SRBC (Holsapple, 1995). Other modifications of the plaque assay can be used to determine effects on immune response to T-cell-independent immunogen (Holsapple, 1995).

\section{Endocrine Modulation}

The potential for chemicals to interfere with endocrine processes, especially those involved in reproduction and development, is the subject of ongoing scientific and regulatory concern. The most sensitive and informative signals of this potential can be derived from studies performed to evaluate systemic toxicity and reproductive and developmental effects. Endpoints capable of detecting endocrine-related effects in these studies include histopathological evaluation of gonads and sexual accessory organs, thyroid, pituitary, and adrenals; male and female fertility; reproductive timing and outcomes; and development of the offspring (e.g., measurement of anogenital distance, preputial separation, vaginal patency, nipple/areolae retention, estrous cycling, sperm evaluation, gross examination of reproductive organs and other tissues, and histopathological evaluation).

The 28-day rat study, a core Tier 1 study as proposed in this approach, is conducted on all compounds to provide a basis for hazard characterization. As such, this study provides an early opportunity to evaluate the potential of substances to interact with endocrine-related processes. Therefore, in addition to the standard organ/tissue weights and histopathology required by the current test guidelines for this study (U.S. EPA, 2000; OECD,
1995), it is recommended that additional organ weights and histopathology of tissues that are presently under consideration in the "Enhanced" OECD 407 test guideline be included (OECD, 2000) (e.g., weight of testes, seminal vesicles and coagulating glands, prostate, ovaries, thyroid, and uterus; and histopathology of pituitary, vagina, epididymides, and mammary gland). In addition, consideration may be given to the inclusion of estrous cyclicity and spermatology (number and morphology) and the retention of serum samples for possible hormonal analyses (e.g., T3, T4, TSH) pending study findings. Not only does the evaluation of these endpoints in the 28-day toxicity study provide an initial assessment of the potential of a chemical to interfere with various endocrinological processes, but the information obtained in this regard may have particular bearing on the subsequent design of further studies to evaluate systemic toxicity or reproduction and development.

The Tier 1 extended one-generation reproduction study in rats proposed by the ACSA Life Stages Task Force (Cooper et al., 2006) also includes a comprehensive evaluation of hormonally mediated endpoints as they pertain to reproduction, growth, and development. The endpoints included are consistent with recent revisions of the U.S. EPA OPPTS 870.3800 test guidelines (U.S. EPA, 1998c) and OECD 416 test guidelines (OECD, 2001), and those that have been more recently proposed for the assessment of endocrine-modulating compounds. The study design also includes the retention of three male and three female F1 offspring per litter, all of which are monitored for the onset of sexual maturity and undergo a gross pathological examination at necropsy, thereby addressing concerns regarding the potential sensitivity of the multigenerational reproduction study to detect low-incidence reproductive-tract malformations.

Changes in one or more parameters in Tier 1 studies would usually lead to more focused investigations targeting potential endocrine mechanisms. Endocrine effects are usually attributable to either direct effects at an endocrinological receptor or effects on the activity of one or more enzymes involved in the production and regulation of hormone levels. Assays such as the Hershberger assay, the uterotrophic assay, and estrogen/androgen receptor-binding assays are useful for identifying compounds with the potential to cause effects by action at the receptor level. Likewise, in vitro assays that assess steroid biosynthesis and detailed hormonal analyses in whole animals are useful in identifying substances with the potential to modulate hormone production. These assays could be performed as second tier studies if indications of hormonally mediated effects are observed. Ultimately, mechanistic investigations should identify the affected receptor or pathway involved, as such information could be useful in refining the risk assessment and extrapolation to humans.

\section{Genetic Toxicity}

The current genotoxicity testing strategies include four core assays: 
- Bacterial mutation assay (Ames test).

- Mammalian cell mutation assay (L5178Y TK assay; CHO HPRT).

- In vitro cytogenetic assay (usually metaphase analysis; human peripheral lymphocytes; CHO; CHL).

- Bone-marrow cytogenetic assay (usually micronucleus assay; rat; mouse).

The Task Force endorses a weight-of-evidence approach to the evaluation of genotoxicity data, and recommends that further developments in this specialized area be incorporated into the safety assessment as they become generally accepted.

\section{Carcinogenicity}

The Task Force considered new experimental models for carcinogenicity involving the use of transgenic mice. A multilaboratory research program coordinated by HESI was conducted between 1996 and 2001 (Robinson and MacDonald, 2001). Importantly, specific criteria for evaluation of the studies were established at the outset in order to permit reliable comparisons to be made of the results obtained from studies performed in multiple laboratories (Popp, 2001). This project was aimed at better characterizing the responsiveness of several alternative models proposed for use in carcinogenicity assessments, including the rasH2 and p53 +/ - models. Alternative, genetically engineered mouse models appear to have usefulness as hazard identification screening models. However, in isolation, these models do not provide reliable evidence to assess potential human carcinogenicity (Cohen et al., 2001). They are not designed to be employed as stand-alone assays; the results obtained from the alternative models need to be evaluated in conjunction with other basic toxicologic information (e.g., toxicokinetics, metabolism, genotoxic potential, doses to which humans are likely to be exposed, etc.) in an overall weight-of-evidence approach (Cohen, 2001; Goodman, 2001). Both the rasH2 and p53 +/- models were considered to be potentially useful when employed as a component of an overall safety assessment (Cohen, 2001; Goodman, 2001; Storer et al., 2001; Usui et al., 2001).

Pritchard et al. (2003) examined existing data on the use of transgenic mouse models for identification of human carcinogens. The focus was on the most extensively used of these mice - including the ras $\mathrm{H} 2$ and p $53+/$ - models - and their performance was compared with the standard 2-year bioassay. The results obtained from the models were used alone and in combination. Data on 99 chemicals were evaluated, including results from the HESI program (Robinson and MacDonald, 2001). The authors made no interpretative conclusions concerning study results, and, for assessments of possible human cancer risk, the conclusions reached by the International Agency for Research on Cancer (IARC) and the National Toxicology Program's Report on Carcinogens (ROC) were accepted. The combined strategy using the $\mathrm{p} 53+/-$ model (for genotoxic compounds) plus the rasH2 model (for both genotoxic and nongenotoxic compounds) made the "correct" call (positive for carcinogens and negative for noncarcinogens) for $88 \%$ of the chemicals. For comparison, identical analysis of chemicals in this dataset that were tested in the standard 2-year, two-species rodent bioassay yielded "correct" calls for $69 \%$ of the chemicals. Although the alternative models had a high percentage of correct calls, they did miss five chemicals that IARC and/or ROC identify as known or probable human carcinogens. The standard bioassay missed none of these compounds; however, it did yield a substantial number of false positives.

It is instructive to examine the five IARC and/or ROC compounds that were reported as "missed" by the rasH2/p53 +/combination in the Pritchard et al. (2003) analysis:

- Cyclosporin A: A potent immunosuppressive. The mechanism of carcinogenic action is very likely due to its pharmacologic action. Though a nongenotoxic compound, it is positive in the p53 +/- model; thus, it was missed in the Pritchard et al. analysis because the investigators limited the p $53+/-$ model to genotoxic compounds.

- Estradiol 17 $\beta$ : Stimulates cell proliferation in sensitive cell populations.

- Phenacetin: Human urothelial tumors arise from chronic abuse, with a proliferative response to chronic cytotoxicity and necrosis. One report by Yamamoto et al. (1998) indicates that it is positive in the rasH2 model.

- Phenobarbital: Causes hypertrophy and hyperplasia in rodent liver. Epidemiology data indicate no evidence for carcinogenicity in humans.

- Chloroform: High doses cause chronic toxicity, necrosis, and compensatory hyperplasia in rodent liver and kidney.

Even if the carcinogenic potential of these compounds had not been detected in other studies, their toxicity would have been detected, resulting in health-protective RfDs that also protect against carcinogenicity.

In all evaluations of the carcinogenicity of agricultural chemicals, each compound is the subject of a genotoxicity evaluation. It is assumed that only compounds viewed as having no significant genotoxic potential will continue in development.

The Task Force supports the view that the conventional mouse bioassay does not add significant additional information for carcinogenicity evaluation over and above the use of the 24-month carcinogenicity assay in the male and female rat (Battershill and Fielder, 1998; Cohen, 2004; Monro, 1993; Usui et al., 1996; Van Oosterhout et al., 1997). The Task Force agrees that the 24-month rat carcinogenicity assay is adequate to detect carcinogens, including those which only cause carcinogenicity following prolonged perturbation of physiological mechanisms. As such, it is the most appropriate assay for those compounds with prolonged exposure, that is, over 6 months. In these cases, the 24-month carcinogenicity assay should be combined with the 12-month rat study described earlier. The evaluation of the potential to cause cancer should involve the consideration of the 
TABLE 6

Final proposed ACSA systemic toxicity study grid

\begin{tabular}{lcc}
\hline $\begin{array}{c}\text { Human exposure } \\
\text { duration }\end{array}$ & $\begin{array}{c}\text { Systemic } \\
\text { toxicity study }\end{array}$ & Carcinogenicity \\
\hline 24 hours or less & $\begin{array}{c}\text { Single-dose rat } \\
\text { or dog }\end{array}$ & Genotoxicity battery \\
2 to 28 days & $\begin{array}{c}\text { 28-day rat or } \\
\text { 90-day dog }\end{array}$ & Genotoxicity battery \\
1 to 6 months & $\begin{array}{c}\text { 90-day dog or } \\
\text { 28-day rat }\end{array}$ & Genotoxicity battery \\
Over 6 months & 12-month rat or & Genotoxicity battery, \\
& 90-day dog & 24-month rat \\
\hline
\end{tabular}

complete database, including evidence of chronic toxicity and determination of modes of toxicity presumed to occur in humans, not just the result of any single study. Ongoing work by HESI and others on the identification and evaluation of the modes of action of chemical carcinogens may lead to approaches for the assessment of carcinogenicity by alternative means other than lifetime studies in rodents. The Task Force recommends that, if and when such approaches become available, they should be adopted for the evaluation of agricultural chemicals as part of the tiered approach proposed here.

When human exposure is of shorter duration, that is, 1 to 6 months, the situation is less clear. In practice, the number of compounds for which this can be assured is small, given the wide variety of uses of most compounds. A need exists for an assay that will detect those compounds that are capable of causing carcinogenicity following a short exposure of 1 to 6 months, even if the latency period before observing carcinogenicity is lengthy. The most obvious mechanism by which this could occur is via genotoxicity, and there is no evidence that there is a need to detect other mechanisms. The battery of in vitro and in vivo models that is currently used should be adequate to detect potential genotoxic carcinogens among the small group of compounds with exposure durations of less than six months with limited, non-domestic use. Any other mechanism will almost certainly involve a response secondary to physiological perturbation, e.g., DES, and this should be readily detected in the proposed shorter-term studies. Additional toxicity data from studies with longer exposure durations will also be available from the life stages studies (Cooper et al., 2006). See Table 6 for the Task Force's proposed final systemic toxicity study grid.

\section{Role of ADME in Assessing Systemic Toxicity}

The most pragmatic route of exposure to be used in the core studies is the oral route. This route is obviously of direct relevance to dietary exposure. Although most operator exposure is via the dermal route, with some contribution from inhalation, there are confounding technical issues in performing dermal studies. Dosimetry is difficult, with much of the applied dose remaining on the occlusive dressing. Animal welfare issues also exist. For example, bandaging animals for extended periods of time can cause significant distress. To design and interpret such studies for maximum utility for routes other than the oral route, knowledge of the absorption, metabolism, distribution, excretion, and kinetics (ADMEK) of the chemical is required. A basic exploration of the ADMEK of a chemical should be conducted before the commencement of core studies. Assessment of the toxicokinetics of agricultural chemicals is reviewed by the ACSA ADME Task Force in Barton et al. (2006). The data can then be used in dose selection and to estimate measures of internal dose. Special care should be taken when extrapolating between exposure durations for chemicals that accumulate or have long half-lives.

Studies will be required to understand the absorption of the chemical by other relevant routes such as dermal and inhalation, and whether any route-specific metabolism is to be expected. These data can then be used in conjunction with the results of the core toxicity studies in which the toxicity is related to the internal dose rather than the administered oral dose to provide hazard characterization for risk assessment. These studies will also demonstrate whether there are any significant effects on the lung or skin caused by direct administration of the chemical.

Therefore, it should be the aim of the overall systemic toxicity evaluation to determine an "internal dose" for each exposure duration that can be used to set an RfD for risk assessment. This dose can then be used to assess the potential effect of each route of exposure by using the specific absorption and kinetic data for each route. The "external" exposure dose will be determined by the various methods of estimating exposure that are currently used or that will be developed in the future. They will then be converted to the "internal" dose using route-specific information. In this way, multiple routes of exposure can also be accommodated.

\section{Incorporation of In Vitro Methodology and New Technologies in Systemic Toxicity Assessment}

The chemicals that will be assessed using the proposed tiered approach devised by the Task Force will be the "survivors" of rigorous selection processes within agricultural chemical companies. In such a selection process, the first evaluation is for the efficacy in providing an effect useful to agriculture. Once efficacy has been established, the potential to cause adverse health and environmental effects is examined. At this stage, many companies employ sophisticated screening and evaluation batteries that include consideration of structure activity and in vitro assays, such as cell culture methods for cytotoxicity, embryo culture methods for teratogenicity, in vitro metabolism, and receptorbinding assays for neurological and endocrinological activity. No company would embark on an expensive development program for a new chemical unless it is confident that there would be a high chance of success. It is difficult to know how these tests which are currently used for selection purposes could be further developed to give definitive information on safety without the need for extensive in vivo studies. This, however, should be a long-term goal of the regulatory scientific community. 
To promote this technical goal, there is increasing interest in utilizing novel technologies derived from computational chemistry, molecular biology, and systems biology for toxicological risk assessment. This new area is referred to as "computational toxicology." In assessing risk associated with exposure to a chemical, there are a number of uncertainties that lie along a continuum, beginning with the presence of the chemical in the environment, the uptake and distribution of the chemical in the organism, the presence of the active chemical at a systemic target site, and the series of biological events that lead to the manifestation of an adverse outcome that can be used for risk assessment. The goal of computational toxicology is to use emerging technologies to improve quantitative risk assessment and reduce uncertainties. The objective includes gathering data on chemical transformation and metabolism, prognostic molecular markers, improved dose metrics, characterization of toxicity pathways, metabonomics, system biological approaches, and modeling frameworks and uncertainty analysis. Computational toxicological approaches may help design studies by predicting potential adverse effects of concern and adding relevant endpoints into the Tier 1 studies.

The approaches that currently hold the greatest potential for assisting in screening new active ingredients and identifying what potential toxicity pathways may be of greatest concern for a new compound are genomics, proteomics, and metabonomics. Defining toxicity pathways associated with initiating events enables one to associate endpoints through multiple levels of biology and, ultimately, to an adverse outcome. Demonstration that toxicity pathways are similar across species and within chemical classes would reduce uncertainty associated with extrapolation across species, and potentially decrease the number of studies required to determine the spectrum of adverse health effects. In the early phase of this developing technology, the greatest benefit appears to be in helping to explain how adverse effects may develop, identifying or confirming a suspected mode of action, and, in species extrapolation, by showing concordance of mechanisms between laboratory animals and humans.

New technological advances hold promise in the development of high throughput screens. These rapid and efficient approaches are a means to provide preliminary effect data on chemicals being considered for further testing. They also have the potential to make significant advances over traditional toxicity testing by increasing the rate of throughput, sensitivity, reproducibility, and reduction in animal use. In brief, these new technologies would include both in vivo and in vitro biological systems in which the active ingredient is tested, RNA and/or protein isolated from the cell culture or target tissues, and subsequent genomic or proteomic assays utilized to identify markers of toxicity that suggest the potential for particular adverse health effects to occur.

\section{Role of Human Data in Risk Assessment}

Human in vivo data, for the most part, will not be available to aid in the design and interpretation of the animal studies that are the focus of the Task Force's proposed tiered testing approach.
When available, however, and derived from studies that meet rigorous scientific and agreed-on ethical standards, human data can provide valuable information to refine risk assessments, especially when there is a clear difference in metabolism and/or kinetics between the animal test species that results in different sensitivity or susceptibility to the compound. ADME studies in humans at less than the LOAEL or even at the NOAEL can provide guidance on which experimental species may be the more relevant to humans (NRC, 2004), and in some cases can provide the basis for the derivation of chemical-specific adjustment factors (CSAFs) (IPCS, 2001). Similarly, human in vitro data, particularly on kinetics and metabolism, may assist in study interpretation and in the development of CSAFs. In some cases, human data have been used to derive RfDs, which are lower than the RfDs derived from animal data (Dourson et al., 2001).

The use of human data is currently an area of active debate. Neither the United States nor the European Commission (EC) has yet formulated policies concerning the use of human data, although both are considering the issue carefully. For example, the U.S. EPA recently issued "a plan to establish a comprehensive framework for making decisions about the extent to which it will consider or rely on certain types of research with human participants" (U.S. EPA, 2005). The Task Force recommends that scientists, risk assessors, and decision-makers stay informed about evolving policies and guidelines regarding the use of human data in risk assessment.

\section{Number of Animals Used in the Proposed Tiered Approach Versus the Current Paradigm}

The total number of animals used in the current core testing paradigm for systemic toxicity has been estimated and is shown in Table 7. Of course, additional animals are used for assessment of acute lethality, skin and eye irritation, skin sensitization, and short-term genotoxicity tests. The number of animals used for these latter studies will not be any different in the proposed tiered testing strategy compared with current practice. A total of 1272 experimental animals is needed for the current core studies.

The following examples illustrate likely animal use following the introduction of the proposed tiered testing approach.

The study requirements and numbers of experimental animals needed are shown in Table 8. For the 28-day rat study, the protocol assumes three treated groups with satellite and recovery groups at all dose levels in both the main study and the satellite groups. For the 90-day dog study, the protocol assumes three treated groups of six per sex per group. The 24-month rat study includes an interim sacrifice at 12 months for assessment of chronic toxicity.

A total of 768 experimental animals is needed for the proposed tiered testing approach. The biggest savings in animal use arises from the elimination of the mouse carcinogenicity bioassay. A comparison of the estimated numbers of animals used in the current core studies versus those used in the core studies of the proposed tiered testing approach are presented in Table 9. 
TABLE 7

Estimated total number of animals used in the current core testing paradigm for systemic toxicity

\begin{tabular}{lccc}
\hline & $\begin{array}{c}\text { Number of } \\
\text { experimental } \\
\text { groups }\end{array}$ & $\begin{array}{c}\text { Number/ } \\
\text { sex/ } \\
\text { group }\end{array}$ & $\begin{array}{c}\text { Total } \\
\text { required }\end{array}$ \\
Study & & & \\
\hline Current studies in the rat & 4 & 5 & 40 \\
$\quad 28$ day & 4 & 10 & 80 \\
90 day & 4 & 10 & 80 \\
Acute neurotox & 4 & 10 & 80 \\
90 -day neurotox & 4 & 50 & 400 \\
24 month & & & 680 \\
Total rats & 4 & 5 & 40 \\
Current studies in the mouse & & 10 & 80 \\
28 day & 4 & 50 & 400 \\
90 day & & & 520 \\
18 month & & & \\
$\quad$ Total mice & 4 & 1 & 8 \\
Current studies in the dog & & 4 & 32 \\
28 day/6 week & 4 & 4 & 32 \\
90 day & 4 & & 72 \\
12 month & & & \\
$\quad$ Total dogs \\
Total number of animals used in current studies & \\
$\quad$ Total (rats, mice, dogs) & & & 1272 \\
\hline
\end{tabular}

\section{THE PROPOSED TIERED TESTING APPROACH}

The Appendix describes the design and specifications of the key systemic toxicity studies in the proposed ACSA tiered testing approach. Successful operation of the approach will demand the use of judgment at a number of key steps as described next.

\section{Step 1: Consider Existing Knowledge}

When embarking on the evaluation of a new agricultural chemical, it is important to consider what information is already known. Such information will include structure-activity relationships for members of a chemical series, the mode of ac-

\section{TABLE 8}

Estimated total number of animals used in the core studies of the proposed ACSA tiered testing approach for systemic toxicity

\begin{tabular}{lccr}
\hline & $\begin{array}{c}\text { Number of } \\
\text { experimental } \\
\text { groups }\end{array}$ & $\begin{array}{c}\text { Number/sex/ } \\
\text { group }\end{array}$ & $\begin{array}{c}\text { Total } \\
\text { required }\end{array}$ \\
\hline Single-dose rat & 4 & 5 & 80 \\
28-day rat & 4 & 20 & 160 \\
90-day dog & 4 & 6 & 48 \\
24-month (including & 4 & 60 & 480 \\
$\quad$ 12-month interim) & & & 768 \\
Total animals & & & \\
\hline
\end{tabular}

TABLE 9

Estimated total number of each species of experimental animals used in the core studies of the proposed ACSA tiered testing approach for systemic toxicity

\begin{tabular}{lcc}
\hline Animals & Current paradigm & New paradigm \\
\hline Rats & 680 & 720 \\
Mice & 520 & 0 \\
Dogs & 72 & 48 \\
Total & 1272 & 768 \\
\hline
\end{tabular}

tion for members of a mechanistic series, and data that may have been generated in the selection process. These data will suggest to the investigator whether there are any special features that should be included in the subsequent evaluation, such as particular modes of action that would require specific assessments (e.g., measurement of cholinesterase inhibition for organophosphates or measurement of plasma tyrosine levels for HPPD inhibitors). The first data to be generated in the formal evaluation are ADME data in the rat. ADME data will help in the design of the core studies by identifying compounds with specific properties (for example, chemicals with particularly long half-lives such as anticoagulants). In addition, all compounds will have assessments of oral and dermal acute toxicity, dermal and ocular irritation, sensitization, and genetic toxicity.

\section{Step 2: Perform the 28-Day Rat Study}

The 28-day rat study will be performed taking into account information from Step 1. The doses are set using information from preliminary studies, such as those that may have been conducted during the selection process and toxicokinetics data that will have been generated before determination of final doses. The data for the indicators of neurotoxicity, endocrine modulation, and immunotoxicity should be considered to determine if second-tier studies are needed to further characterize any effects.

\section{Step 3: Perform the 90-Day Dog Study}

The 90-day dog study is performed taking into account information from Step 1. The doses are set on the basis of a preliminary dog study, which should also include an assessment of kinetics by both the oral and dermal routes.

\section{Step 4: Consider the Relative Sensitivity and Susceptibility of the Rat and Dog}

The results of the 28-day rat and the 90-day dog studies should be compared to determine relative susceptibility (i.e., what effects occurred) and relative sensitivity (i.e., at what dose levels the effects occurred). The purpose is to identify the species that will be used to provide guidance for human health protection. In the absence of information to the contrary, the species chosen will be the one that provides the lower RfD. Effects in this species are used to determine the relevant uncertainty factor, which is then applied to the NOAEL. Note that the rat study will have been conducted for a shorter duration than the dog study. 
Therefore, it would be expected that the rat 28-day NOAEL may be higher for compounds with cumulating kinetics or for those causing effects that become more severe with repeated insult or injury. As a rule of thumb, the rat 28-day NOAEL could be divided by a factor of 3 before being compared with the dog 90-day NOAEL. To the extent possible, the comparison of dose levels should be based on knowledge gained from toxicokinetics, which elucidates internal, rather than applied, dose. The conservative assumption that the more sensitive species is more relevant can also be confirmed or rebutted on the basis of additional scientific knowledge.

\section{Step 5: Derive the RfD for Human Exposure Durations Up to 6 Months}

Scenario 1: The Rat is the "Relevant" Species

Human exposure durations of 2 to 28 days should be considered by deriving an RfD from the 28-day rat study by dividing the NOAEL by an appropriate uncertainty factor.

Human exposure durations of up to 1 day should be considered by deriving an RfD from an endpoint from the 28-day rat study relevant for acute exposure by dividing the NOAEL by an appropriate uncertainty factor. If the RfD derived from the 28-day rat study indicates an adequate margin of exposure, then the requirement to perform a single-dose study could be waived. However, the decision to perform a single-dose study often needs to be taken early in the development of an agricultural chemi$\mathrm{cal}$, that is, before the chemical's use rate and all of its potential outlets have been determined. This may limit the opportunity to waive the single-dose study on exposure grounds. In addition, the single-dose study is valuable in providing insight into kinetics (speed of onset, recovery period) of the effect caused by the compound. This information can be used to assess intermittent and variable exposure as described in Step 6. For these reasons, it is expected that a single-dose study will be performed more often than not.

Human exposure durations of 1 to 6 months should be considered by deriving an RfD by dividing the NOAEL by an appropriate uncertainty factor from the 90-day dog study if the two species are considered to be of equal sensitivity. If the rat is more sensitive, then the results of the 28-day rat study should be used with an additional factor of 3 .

\section{Scenario 2: The Dog is the "Relevant" Species}

Human exposure durations of 2 to 28 days should be considered by deriving an RfD from the 90-day dog study by dividing the NOAEL by an appropriate uncertainty factor.

Human exposure durations of up to 1 day should be considered by deriving an RfD from an endpoint from the 90-day dog study relevant for acute exposure by dividing the NOAEL by an appropriate uncertainty factor, or by considering the results of the first day of dosing if the effect can be determined by the recorded parameters. If the RfD derived from the 90-day dog study indicates an adequate margin of exposure, then the requirement to perform a single-dose study could be waived. However, the decision to perform a single-dose study often needs to be made early in the development of an agricultural chemical, that is, before the chemical's use rate and all of its potential outlets have been determined. This may limit the opportunity to waive the single-dose study on exposure grounds. In addition, the single-dose study will be valuable in providing insight on the kinetics (speed of onset, recovery period) of the effect caused by the compound. This information can be used to assess intermittent and variable exposure as described in Step 7. For these reasons, it is expected that a single-dose study will be performed more often than not.

Human exposure durations of 1 to 6 months should be considered by deriving an RfD by dividing the NOAEL by an appropriate uncertainty factor from the 90 -day dog study.

\section{Step 6: Derive the RfD for Human Exposure Durations Over 6 Months}

Scenario 1: The Rat is the "Relevant" Species

If there is human exposure duration of greater than 6 months, then a 24-month rat study with a 12-month interim sacrifice should be performed to assess chronic toxicity and carcinogenicity. An RfD for chronic toxicity should be derived by dividing the 12-month interim sacrifice NOAEL by an appropriate uncertainty factor. The results of the 24-month phase of the study should be used to assess carcinogenicity. Subsequent classification and risk assessment of the compound would then be done following established national and regional policy. If a significant nonneoplastic effect emerges only in the second year of the study, then it should be taken into account when deriving a lifetime RfD.

\section{Scenario 2: The Dog is the "Relevant" Species}

If the duration of human exposure is likely to be greater than 6 months, then a 24-month rat study with a 12-month interim sacrifice should be performed to assess chronic toxicity. An RfD should be calculated both from the rat study and the 90-day dog study. This should be done taking into account the nature of the effects observed. In the absence of information to the contrary, the lower RfD should be used for risk assessments for exposure durations of over six months. The results of the 24-month phase of the rat study should be used to assess carcinogenicity. Subsequent classification and risk assessment of the compound would then be done following established national and regional policy.

\section{Step 7: Derivation of RfDs for Varying and/or Intermittent Exposures}

Although many risk assessments assume a constant exposure level throughout the duration of exposure, in most cases the exposure levels do vary. The question arises as to how these variations should be considered. Should a time-weighted average be used over the entire exposure period no matter what the day-to-day variation may be, or should the RfD not be exceeded for any given day? This question can only be addressed with certainty when the toxicokinetics of the compound and the kinetics of the effect are taken into account. 
TABLE 10

NOAELs from 90-day rat, 24-month rat, 90-day dog, and 12-month dog studies compared with the lowest NOAEL excluding the 12-month dog study (SABRE data)

\begin{tabular}{|c|c|c|c|c|c|c|c|}
\hline $\begin{array}{l}\text { Active } \\
\text { ingredient }\end{array}$ & $\begin{array}{l}\text { Chemical } \\
\text { class }\end{array}$ & $\begin{array}{l}\text { 90-Day rat } \\
\text { NOAEL } \\
\text { (mg/kg/day) }\end{array}$ & $\begin{array}{l}\text { 90-Day dog } \\
\text { NOAEL } \\
\text { (mg/kg/day) }\end{array}$ & $\begin{array}{c}\text { 1-Year } \\
\text { dog NOAEL } \\
\text { (mg/kg/day) }\end{array}$ & $\begin{array}{c}\text { 2-Year rat } \\
\text { NOAEL } \\
\text { (mg/kg/day) }\end{array}$ & $\begin{array}{l}\text { Lowest } \\
\text { NOAEL } \\
\text { all studies }\end{array}$ & $\begin{array}{l}\text { Lowest NOAEL } \\
\text { excluding } \\
\text { chronic dog }\end{array}$ \\
\hline 2,4-D & Phenoxyacid & 15 & 1 & 1 & 5 & 1 & 1 \\
\hline Acetochlor & Acetanilide & 80 & 10 & 2 & 10 & 2 & 10 \\
\hline Atrazine & Triazine & 1 & 6 & 5 & 3.5 & 1 & 1 \\
\hline Butylate & Carbamate & 32 & 45 & 5 & 100 & 5 & 32 \\
\hline Carbaryl & Carbamate & 125 & 1 & $\begin{array}{c}3.1 \\
\text { (LOAEL) }\end{array}$ & 10 & 1 & 1 \\
\hline Cyprodinil & Anilinopyrimidine & 3 & 46 & - & 2.7 & 2.7 & 2.7 \\
\hline Diazinon & Organophosphorothioate & $\begin{array}{c}0.3 \\
\text { (LOAEL) }\end{array}$ & $\begin{array}{c}0.02 \\
\text { (LOAEL) }\end{array}$ & 0.004 & 20 & 0.004 & $\begin{array}{c}0.006 \\
\text { (LOAEL/3) }\end{array}$ \\
\hline $\begin{array}{l}\text { Glufosinate } \\
\text { ammonium }\end{array}$ & $\begin{array}{l}\text { Phosphinic analogue of } \\
\text { glutamic acid }\end{array}$ & $\begin{array}{c}3.2 \\
\text { (LOAEL) }\end{array}$ & 2 & 5 & 2 & 2 & 2 \\
\hline Hexaconazole & Triazole & 3 & 5 & 2 & 4.7 & 2 & 3 \\
\hline Mevinphos & Organophosphate & $\begin{array}{c}0.25 \\
\text { (LOAEL) }\end{array}$ & $\begin{array}{c}0.0625 \\
\text { (LOAEL) }\end{array}$ & 0.025 & 0.025 & 0.025 & 0.025 \\
\hline Prallethrin & Pyrethroid & 24 & 3 & 5 & 16.3 & 3 & 3 \\
\hline Tebuconazole & Triazole & 9 & 73 & 2.9 & 5 & 2.9 & 5 \\
\hline Triallate & thiocarbamate & 3 & 2 & 1.275 & $\begin{array}{c}\text { NA } \\
(0.5=\text { LOAEL })\end{array}$ & $\begin{array}{c}0.5 \\
\text { (LOAEL) }\end{array}$ & $\begin{array}{c}0.5 \\
\text { (LOAEL) }\end{array}$ \\
\hline Vinclozoline & Dicarboximide & 4 & 3 & 2.4 & 1 & 1 & 1 \\
\hline Dicamba & Dichlorobenzoate & 250 & 6 & 52 & $\geq 125$ & 6 & 6 \\
\hline Dimethoate & Organophosphate & 2 & $<0.25$ & $<0.18$ & 0.05 & 0.05 & 0.05 \\
\hline Chlorfenapyr & Pyrrole & 24 & 4 & 4 & No study & 4 & 4 \\
\hline MCPA & Dithiocarbamate & 7 & 3 & 1.75 & 4.38 & 1.75 & 3 \\
\hline Metolachlor & Acetanilide & 23 & 10 & 9.7 & 15 & 9.7 & 10 \\
\hline Benomyl & Benzimidazole & 25 & 13 & 12.5 & $>125$ & 12.5 & 13 \\
\hline Propachlor & Acetanilide & 75 & 38 & 6.25 & 2.4 & 2.4 & 2.4 \\
\hline 2,4-DB & Phenoxyacid & 16 & 8 & $<2.39$ & 3 & 2.39 & 3 \\
\hline Fosetyl-AL & Organophosphate & 365 & 274 & 250 & 400 & 250 & 274 \\
\hline Iprodione & Dicarboximide & 78 & 60 & 17.5 & 6.1 & 6.1 & 6.1 \\
\hline Bifenthrin & Pyrethroid & 3 & 2 & 1.3 & 2.5 & 1.3 & 2 \\
\hline Carboxin & Oxathin & 6 & 8 & No study & 0.8 & 0.8 & 0.8 \\
\hline Dicofol & organochlorine & $\begin{array}{c}0.64 \\
\text { (LOAEL) }\end{array}$ & $\begin{array}{c}2.5 \\
\text { (LOAEL) }\end{array}$ & 0.12 & 1 & 0.12 & $\begin{array}{c}0.2 \\
(\mathrm{LOAEL} / 3)\end{array}$ \\
\hline Lactofen & Diphenyl ether & 14 & 75 & 0.79 & 0.79 & 0.8 & 0.8 \\
\hline
\end{tabular}

For instance, consider a chemical that has a very long halflife such that it accumulates in the body. Adverse effects are observed when the body burden reaches a certain level. It is likely that for such a chemical the RfDs derived from the proposed tiered testing approach would be significantly lower with longer duration of exposure. For such chemicals, it would be sensible to apply the time-weighted average approach.

At the other extreme, consider a chemical that has a short pharmacological, but no lasting, effect. If it also has a short half-life, it is likely that the RfDs derived from the proposed tiered testing approach will be similar no matter what the duration of exposure. In these cases, the individual daily expo- sure should be considered, rather than the cumulative or timeweighted value.

The proposed ACSA tiered testing approach is designed to give information to help characterize the compound. The toxicokinetics package defines the half-life and provides information on whether the chemical is likely to reach a higher level of internal dose as exposure progresses. The single-exposure study and the recovery period in the 28-day rat study give insight into the kinetics of the effects, whether there is recovery and, if so, over what period. A major objective of the single-exposure study is, in fact, to provide this information. Consequently, consideration should be given to performing the single-exposure study. 
The proposed tiered testing approach provides the risk assessor with RfDs for each of the human exposure time periods (1 day, 2 to 28 days, 1 to 6 months, and greater than 6 months). These RfDs can be used to provide guidance on how to assess varying and intermittent exposures in the following way:

- The time-weighted average daily dose for any given portion of the exposure should not exceed the relevant RfD.

To expand this principle:

- No single day's exposure should be above the 1-day RfD.

- The time-weighted average daily exposure for any period of 2 to 28 days should not exceed the 2- to 28 -day RfD.

- The time-weighted average daily exposure for any period of 1 to 6 months should not exceed the 1- to 6months RfD.

- The time-weighted average daily exposure for any period of 6 months should not exceed the over-6-months RfD.

This guidance holds for compounds at both ends of the spectrum, because, as explained earlier, the relationship between the RfDs will reflect their properties.

\section{EVALUATION OF THE PROPOSED TIERED TESTING APPROACH}

The proposed ACSA tiered testing approach differs in two significant ways from the current testing scheme:

- It contains new elements designed to provide more relevant data for deriving RfDs for shorter time periods of human exposure, for example, a single-dose study, a 28-day rat study with recovery period, and kinetics. It is not possible to evaluate the impact of these new studies by using the existing database.

- It contains fewer studies to derive the longer term RfD, that is, neither a 12-month dog study nor a mouse carcinogenicity study is recommended. The SABRE database described earlier has been used to evaluate the theoretical performance of this aspect of the proposed tiered approach. The database contains a set of 28 chemicals for which the majority of relevant study types that comprise the components of the current systemic toxicity testing paradigm for agricultural chemicals are represented. These core studies are the 90-day studies in the rat and dog, the combined chronic and carcinogenicity study in the rat, and the carcinogenicity study in the mouse.

\section{Impact of Removing the 12-Month Dog Study}

Generally, the longer term RfD is derived by applying a safety or uncertainty factor to the lowest NOAEL from the 90-day rat study, the 24-month rat study, the 90-day dog study, and the 12-month dog study. The impact of removing the 12-month dog study has been evaluated by comparing the lowest NOAEL from these four studies with the lowest NOAEL derived from the three studies that remain, if the 12-month dog study is not considered. These studies are summarized in Figure 5 and the results of this evaluation are shown in Table 10 and Figure 6. For only two compounds in the database, acetochlor and butylate, would the lowest NOAEL from the other three studies be more than twice the NOAEL for the 12-month dog study. The data for acetochlor are confounded by the presence of two 12-month dog studiesone with a NOAEL of $2 \mathrm{mg} / \mathrm{kg} / \mathrm{day}$, giving a ratio of 5 , and one with a NOAEL of $12 \mathrm{mg} / \mathrm{kg} /$ day, giving a ratio of 1 . Both values

Ratio of Lowest NOAELs with and without 1-Year Dog

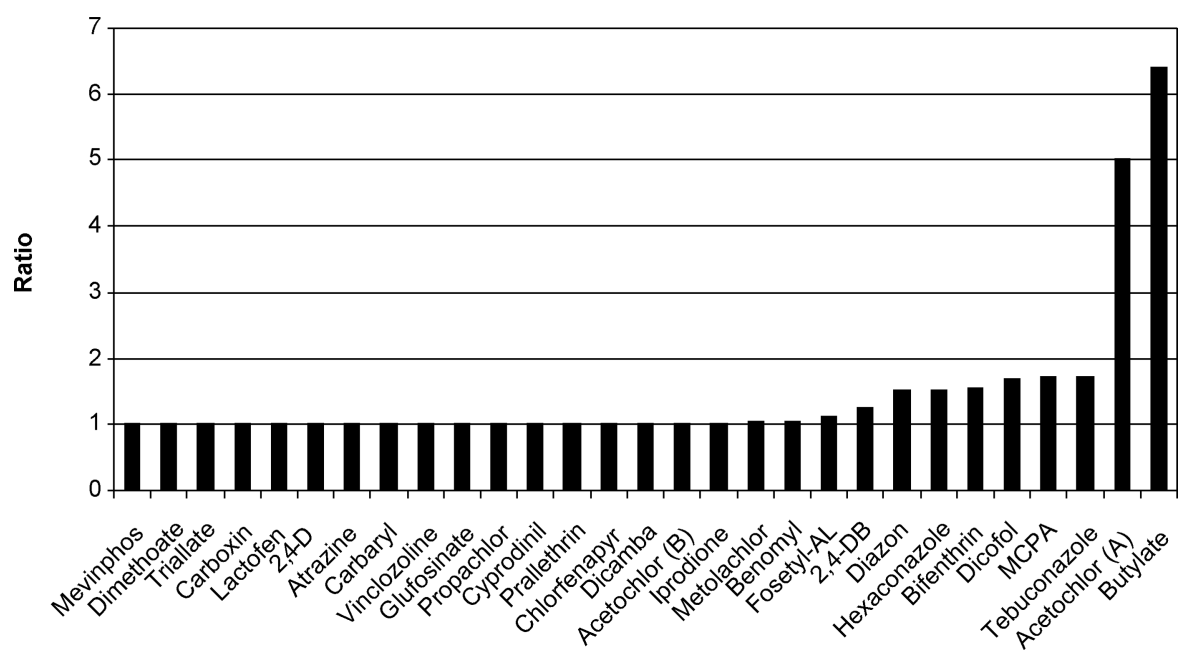

FIG. 6. Ratio of lowest NOAELs from 90-day rat, 24-month rat, 90-day dog, and 12-month dog studies compared with the lowest NOAEL, excluding the 12-month dog study. 
are shown in Figure 6. Butylate is more problematic. The 12month dog study has a much lower NOAEL, and the LOAEL is above the NOAEL for the 90-day dog study; therefore, the difference is unlikely to be caused by a dosing artifact. However, the NOAEL for the 90-day rat study is lower than that for the 24month rat study. The entire database for butylate can be regarded as unusual.

\section{Impact of Removing the Mouse Carcinogenicity Study}

The impact of removing the mouse carcinogenicity study was discussed earlier. To review, tumors were detected in the rat, with 13 of the 27 chemicals remaining in the analysis (six of these also induced tumors in the mouse). An additional four chemicals induced mouse tumors in the absence of findings in the rat; however, all but one of these chemicals resulted in liver tumors (see Tables 4 and 5). The relevance of the modes of action for these mouse liver tumors for human health is questionable.

\section{CONCLUSION}

The proposed tiered testing approach for agricultural chemical safety assessment includes a number of key principles:

- The core 28-day rat study is the most comprehensive initial assessment of toxic potential, and includes satellite groups for the assessment of neuropathology at every dose level, an assessment of recovery/progression of general toxicity at all dose levels, and neuropathology at all dose levels.

- The core 90-day dog study includes an assessment of recovery/progression at all dose levels. The standard study design can be augmented to include satellite groups for specific endpoints such as neuropathology if indicated from the results of preceding studies in either the rat or the dog (e.g., for range finding).

- The 24-month combined chronic toxicity/carcinogenicity study in the rat includes an assessment of chronic toxicity at 12 months, which allows for some assessment of progression beyond 12 months and allows the potential effects of aging to be taken into account.

The proposed tiered testing approach has the potential to provide new information for risk assessment for shorter human exposure durations while reducing the number of animals used and without compromising the sensitivity of the determination of longer-term RfDs. However, it is recognized that the approach to date has been challenged with only a relatively limited number of compounds. It is anticipated that, once disseminated, the approach will be further evaluated by the scientific community using a wider range of compounds. The Task Force encourages investigators engaged in such work to place the results of such evaluations in the public domain.

\section{ACKNOWLEDGMENTS}

The HESI ACSA Technical Committee and the ACSA Systemic Toxicity Task Force appreciate the contributions of Lorraine Irvine (toXcel International Ltd.) and Bernhard Stahl, who served as scientific liaisons from the ACSA Life Stages Task Force and ADME Task Force, respectively. Grateful acknowledgment is given to Chris Sheard (Syngenta CTL) and Dr. Whang Phang (U.S. EPA) for their development and application of the SABRE database.

No conflict of interest is declared.

\section{ABOUT HESI}

The ILSI Health and Environmental Sciences Institute (HESI) is a global branch of the International Life Sciences Institute, a public, nonprofit scientific foundation with branches throughout the world. HESI provides an international forum to advance the understanding and application of scientific issues related to human health, toxicology, risk assessment, and the environment. HESI is widely recognized among scientists from government, industry, and academia as an objective, sciencebased organization within which important issues of mutual concern can be discussed and resolved in the interest of improving public health. As part of its public benefit mandate, HESI's activities are carried out in the public domain, generating data and other information for broad scientific use and application. Further information about HESI can be found at http://www.hesiglobal.org.

\section{REFERENCES}

Barton, H.A., Pastoor, T.P., Baetcke, K., Chambers, J.E., Diliberto, J., Doerrer, N.G., Driver, J.H., Hastings, C.E., Iyengar, S., Krieger, R., Stahl, B., and Timchalk, C. (2006). The acquisition and application of absorption, distribution, metabolism, and excretion (ADME) data in agricultural chemical safety assessments. Crit. Rev. Toxicol. 36:9-35.

Basketter, D.A., Bremmer, J.N., Buckley, P., Kammuller, M.E., Kawabata, T., Kimber, I., Loveless, S.E., Magda, S., Stringer, D.A., and Vohr, H.-W. (1995). Pathology considerations for, and subsequent risk assessment of, chemicals identified as immunosuppressive in routine toxicology. Food Chem. Toxicol. 33:239243.

Battershill, J.M., and Fielder, R.J. (1998). Mouse-specific carcinogens: an assessment of hazard and significance for validation of short-term carcinogenicity bioassays in transgenic mice. Hum. Exp. Toxicol. 17:193-205.

Carmichael, N.G., Barton, H.A., Boobis, A.R., Cooper, R.L., Dellarco, V.L., Doerrer, N.G., Fenner-Crisp, P.A., Doe, J.E., Lamb, J.C., and Pastoor, T.P. (2006). Agricultural chemical safety assessment: A multi-sector approach to the modernization of human safety requirements. Crit. Rev. Toxicol. 36:1-7.

Cohen, S.M. (2001). Alternative models for carcinogenicity testing: Weight of evidence evaluations across models. Toxicol. Pathol. 29(Suppl.):183-190.

Cohen, S.M. (2004). Human carcinogenic risk evaluation: An alternative approach to the two-year rodent bioassay. Toxicol. Sci. 80:225229.

Cohen, S.M., Robinson, D., and MacDonald, J. (2001). Alternative models for carcinogenicity testing. Toxicol. Sci. 64:14-19.

Cooper, R.L., Lamb, J.C., Barlow, S.M., Bentley, K., Brady, A.M., Doerrer, N.G., Eisenbrandt, D.L., Fenner-Crisp, P.A., Hines, R.N., Irvine, L., Kimmel, C.A., Koeter, H., Li, A.A., Makris, S.L., Sheets, 
L., Speijers, G.J.A., and Whitby, K. (2006). A tiered approach to life stages testing for agricultural chemical safety assessment. Crit. Rev. Toxicol. 36:69-98.

Counts, J.L., and Goodman, J.I. (1995). Principles underlying dose selection for, and extrapolation from, the carcinogen bioassay: Dose influences mechanism. Regul. Toxicol. Pharmacol. 21:418-421.

Dourson, M.L., Andersen, M.E., Erdreich, L.S., and MacGregor, J.A. (2001). Using human data to protect the public's health. Regul. Toxicol. Pharmacol. 33:234-256.

European Crop Protection Association. (1999). Relationship between no effect levels and low effect levels in rat 28 day and 90 day studies. Unpublished report.

Gaylor, D.W. (2005). Are tumor incidence rates telling us what we need to know about carcinogens? Regul. Toxicol. Pharmacol. 41:128133.

Goodman, J.I. (2001). A perspective on current and future uses of alternative models for carcinogenicity testing. Toxicol. Pathol. 29(Suppl.): 173-176.

Holsapple, M.P. (1995). The plaque-forming cell (PFC) response in immunotoxicology: An approach to monitoring the primary effector function of B-lymphocytes. In: Methods in Immunotoxicology, Vol. 1, G.R. Burleson, J.H. Dean, and A.E. Munson, eds., pp. 71108, Wiley-Liss, New York.

Huff, J.E., Bucher, J.R., Schwetz, B.A., and Barrett, J.C. (1994). Optimum exposure levels for chemical carcinogenesis experiments: Concepts, principles, guidelines, and experience. Toxicologist, SOT Abstract 475.

International Programme on Chemical Safety. (2001). Guidance document for the use of data in development of chemical-specific adjustment factors (CSAFs) for interspecies differences and human variability in dose/concentration-response assessment. World Health Organization (WHO), Geneva, Switzerland. http://www.who.int/ ipcs/publications/methods/harmonization/en/csafs_guidance_doc. pdf.

Luster, M.I., Munson, A.E., Thomas, P.T., Holsapple, M.P., Fenters, J.D., White, K.L., Jr., Lauer, L.D., Germolec, D.R., Rosenthal, G.J., and Dean, J.H. (1988). Development of a testing battery to assess chemical-induced immunotoxicity: National Toxicology Program's guidelines for immunotoxicity evaluation in mice. Fundam. Appl. Toxicol. 10:2-19.

Luster, M.I., Pait, D.G., Portier, C., Rosenthal, G.J., Germolec, D.R., Comment, C.E., Munson, A.E., White, K., and Pollock, P. (1992a). Qualitative and quantitative experimental models to aid in risk assessment for immunotoxicology. Toxicol. Lett. 64/65:71-78.

Luster, M.I., Portier, C., Pait, D.G., White, K.L., Jr., Gennings, C., Munson, A.E., and Rosenthal, G.J. (1992b). Risk assessment in immunotoxicology I: Sensitivity and predictability of immune tests. Fundam. Appl. Toxicol. 18:200-210.

Luster, M.I., Portier, C., Pait, D.G., Rosenthal, G.J., Germolec, D.R., Corsini, E., Blaylock, B.L., Pollock, P., Kouchi, Y., Craig, W., White, D.L., Munson, A.E., and Comment, C.E. (1993). Risk assessment in immunotoxicology II: Relationships between immune and host resistance tests. Fundam. Appl. Toxicol. 21:71-82.

McClain, R.M. (1994). Mechanistic considerations in the regulation and classification of chemical carcinogens. In: Nutritional Toxicology, F.N. Kotsonis, M. Mackey, and J. Hjelle, eds., pp. 273-304. Raven Press, New York.

McConnell, E. (1989). The maximum tolerated dose: The debate. $J$. Am. Coll. Toxicol. 8:1115-1120.
McConnell, E. (1995). Historical review of the rodent bioassay and future directions. Regul. Toxicol. Pharmacol. 21:38-43.

Mead, R. (1988). The Design of Experiments. Cambridge University Press, Cambridge, UK.

Monro, A. (1993). How useful are chronic (life-span) toxicology studies in rodents in identifying pharmaceuticals that pose a carcinogenic risk to humans? Adverse Drug React. Toxicol. Rev. 12:534.

Moser, V.C., and MacPhail, R.C. (1990). Comparative sensitivity of neurobehavioural tests for chemical screening. Neurotoxicology 11:335-344.

National Research Council. (1993). Use of the maximum tolerated dose in animal bioassays for carcinogenicity. In: Issues in Risk Assessment, B. Goldstein et al., eds., pp. 15-83, National Academies Press, Washington, DC.

National Research Council. (2004). Committee on the Use of Third Party Toxicity Research with Human Research Participants, National Research Council of the National Academies. National Academies Press, Washington, DC.

National Toxicology Program. (1984). Report of the NTP Ad Hoc Panel on Chemical Carcinogenesis Testing and Evaluation. National Institute of Environmental Health Sciences, Research Triangle Park, NC.

National Toxicology Program. (1992). Final report of the advisory review by the NTP Board of Scientific Councilors. Fed.Reg. 57:3172131730.

Organisation for Economic Cooperation and Development. (1981). Health effects test guideline 453. Combined chronic toxicity/carcinogenicity studies. Environment Directorate, Paris, France.

Organisation for Economic Cooperation and Development. (1995). Health effects test guideline 407. Repeated dose 28-day oral toxicity study in rodents. Environment Directorate, Paris, France.

Organisation for Economic Cooperation and Development. (1998). Health effects test guideline 409. Repeated dose 90-day oral toxicity study in non-rodents. Environment Directorate, Paris, France.

Organisation for Economic Cooperation and Development. (2000). Protocol for investigating the efficacy of the Enhanced 407 Test Guideline (phase 2). Rationale for the investigation, and description of the protocol. Environment Directorate, Paris, France.

Organisation for Economic Cooperation and Development. (2001). Health effects test guideline 416. Two-generation reproduction toxicity study. Environment Directorate, Paris, France.

Popp, J.A. (2001). Criteria for the evaluation of studies in transgenic models. Toxicol. Pathol. 29(Suppl.):20-23.

Pritchard, J.B., French, J.E., Davis, B.J., and Haseman, J.K. (2003). Transgenic mouse models: Their role in carcinogen identification. Environ. Health Perspect. 111:444-454.

Robinson, D.E., and MacDonald, J.S. (2001). Background and framework for ILSI's collaborative evaluation program on alternative models for carcinogenicity assessment. Toxicol. Pathol. 29(Suppl.):1319.

Rozman, K.K., and Doull, J. (2000). Dose and time as a variable of toxicity. Toxicology 144:169-178.

Solecki, R. (2001). Pesticide residues in food-2000. Report of the Joint Meeting of the FAO Panel of Experts on Pesticide Residues in Food and the Environment and the WHO Core 
Assessment Group. Annex 5: Proposed test guidelines for studies with single oral doses (for use in establishing acute reference doses for chemical residues in food and drinking water). FAO Plant Production and Protection Paper 163. FAO, Rome, Italy. http://www.fao.org/ag/agp/agpp/pesticid/jmpr/Download/2000_rep/ cont.pdf

Sontag, J.M., Page, N.P., and Safiotti, U. (1976). Guidelines for carcinogen bioassay in small rodents. DHHS Publication (NIH) 76-801. National Cancer Institute, Bethesda, MD.

Spielmann, H., and Gerbracht, U. (2001). The use of dogs as second species in regulatory testing of pesticides. Part II: Subacute, subchronic and chronic studies in the dog. Arch. Toxicol. 75:121.

Storer, R.D., French, J.E, Haseman, J., Hajian, G., LeGrand, E.K., Long, G.G., Mixson, L.A., Ochoa, R., Sagartz, J.E., and Soper, K.A. (2001). P53 +/- hemizygous knockout mouse: overview of available data. Toxicol. Pathol. 29(Suppl.):30-50.

U.S. Environmental Protection Agency. (1998a). Health Effects Test Guideline OPPTS 870.3100. 90-Day oral toxicity in rodents. EPA 712-C-98-199. Office of Prevention, Pesticides and Toxic Substances, Washington, DC.

U.S. Environmental Protection Agency. (1998b). Health Effects Test Guideline OPPTS 870.3150. 90-Day oral toxicity in nonrodents. EPA 712-C-98-200. Office of Prevention, Pesticides and Toxic Substances, Washington, DC.

U.S. Environmental Protection Agency. (1998c). Health Effects Test Guideline OPPTS 870.3800. Reproduction and fertility effects. EPA 712-C-98-208. Office of Prevention, Pesticides and Toxic Substances, Washington, DC.

U.S. Environmental Protection Agency. (1998d). Health Effects Test Guideline OPPTS 870.4300. Combined chronic toxicity/carcinogenicity. EPA 712-C-98-212. Office of Prevention, Pesticides and Toxic Substances, Washington, DC.
U.S. Environmental Protection Agency. (2000). Health Effects Test Guideline OPPTS 870.3050. Repeated dose 28-day oral toxicity study in rodents. EPA 712-C-00-366. Office of Prevention, Pesticides and Toxic Substances, Washington, DC.

U.S. Environmental Protection Agency. (2003a). Draft final guidelines for carcinogen risk assessment. Fed. Reg. 68: 10012.

U.S. Environmental Protection Agency. (2003b). Rodent carcinogenesis studies: dose selection and evaluation. Interim Guidance Document No. G2003.02. Office of Pesticide Programs, Health Effects Division, Washington, DC.

U.S. Environmental Protection Agency. (2005). Human Testing; Proposed plan and description of review process. Fed. Reg. 70: 6661.

Usui, T., Griffiths, S.A., and Lumley, C.E. (1996). Industry viewpoint: The utility of the mouse for the assessment of the carcinogenic potential of pharmaceuticals. In: Proceedings, Third International Conference on Harmonisation, Yokohama, 1995, F. D'Arcy and D.W.G. Harron, eds., pp. 279-284. Greystone Books, Antrim, N. Ireland.

Usui, T., Mutai, M., Hisada, S., Takoaka, M., Soper, K.A., McCullough, B., and Alden, C. (2001). CB6F1-rasH2 mouse: Overview of available data. Toxicol Pathol. 29(Suppl.):90-108.

Van Oosterhout, J.P., Van der Laan, J.W., De Waal, E.J., Olejniczak, K., Hilgenfeld, M., Schmidt, V., and Bass, R. (1997). The utility of two rodent species in carcinogenic risk assessment of pharmaceuticals in Europe. Regul Toxicol. Pharmacol. 25:6-17.

Ware, G.W. (2000). The Pesticide Book, 5th ed., Thomson, Fresno, CA. Yamamoto, S., Urano, K., Koizumi, H., Wakana, S., Hioki, K., Mitsumori, K., Kurokawa, Y., Hayashi, Y., and Nomura, T. (1998). Validation of transgenic mice carrying the human prototype c-Ha-ras gene as a bioassay model for rapid carcinogenicity testing. Environ. Health Perspect. 106(Suppl. 1):57-69.

\section{APPENDIX: DESCRIPTION OF KEY SYSTEMIC TOXICITY STUDIES}

\section{Proposed Design of the Core 28-Day Toxicity Study} in the Rat

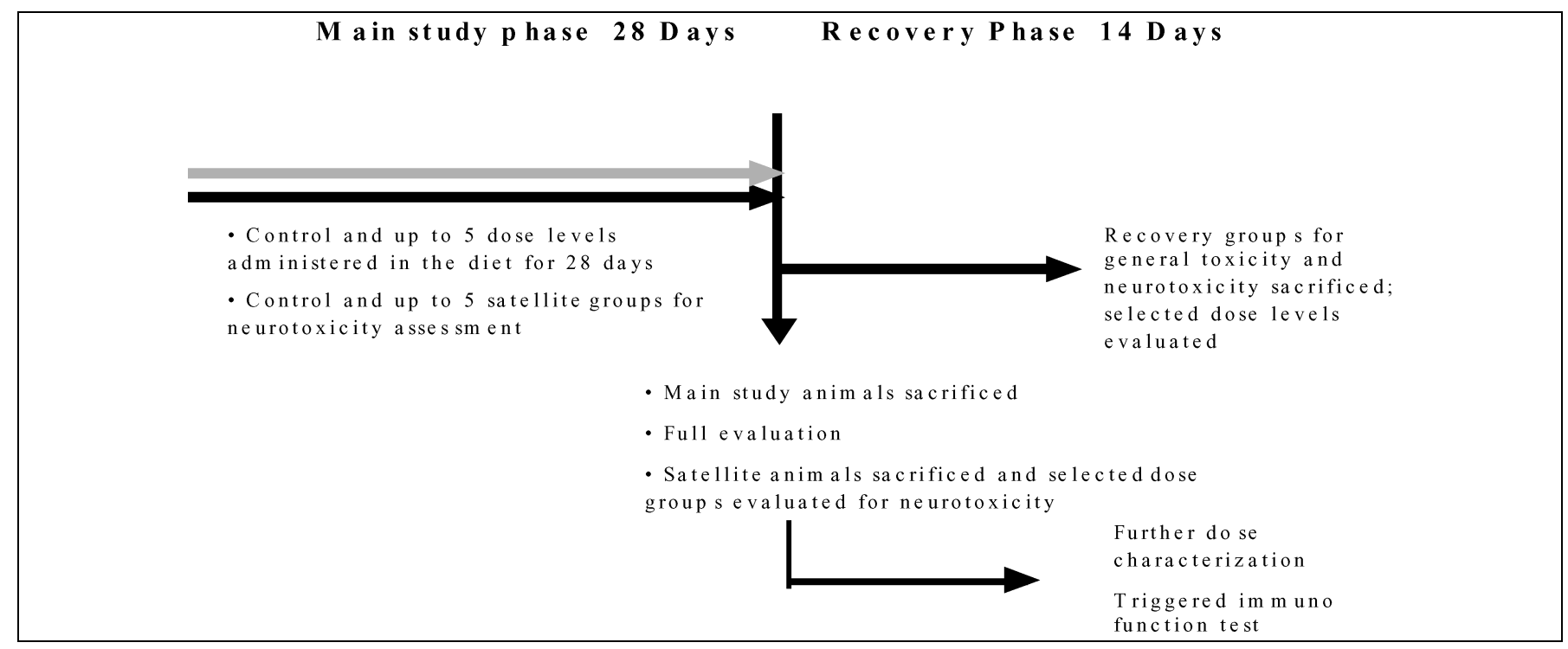


- The proposed 28-day rat study is the pivotal study in the assessment of subchronic toxicity, and is used to provide information on relevance to human exposure durations from 1 day up to 6 months either alone or in conjunction with the 90-day dog study. Implicit in the study design is the need to identify subtle changes, as well as expression of more significant toxicity.

- Dose levels are selected taking into account the outcome of range-finding studies, and are supported by relevant ADME data.

- The numbers of experimental animals used for the main study, satellite groups, and recovery phase are scientifically based on power calculations. For example, and as guidance, power calculations indicate that for liver growth, a minimum of six rats/sex/group (in a 28-day study) are needed to detect a $10 \%$ increase in liver weight, with a power of $80 \%$ and at the $5 \%$ significance level. These numbers are consistent with the outcome of other statistical methods used in study design, such as the resource equation method of Mead (Mead, 1988). According to this method, the errors of freedom $(E)$ in an experiment should lie between 10 and 20-any more may involve the unnecessary use of animals; any fewer may give unreliable results. $E$ is defined (for a completely random design) as (total number of animals) - (number of experimental groups). Therefore, for a 28-day study with four experimental groups using six animals/sex/group, $E=(24-4)=20$, which is within acceptable limits.

\section{Summary of Study Specification-Core 28-Day Toxicity Study in the Rat}

Groups:

Number of animals:

Analysis of diets:

Dose administration:

Clinical observations:

Body weights:

Food consumption:

Functional observational

battery:

Motor activity:

Clinical pathology:

Pathology:
Main study one control and up to five test. Recovery groups for main study and satellite groups at all dose levels.

Main study, recovery and neurotoxicity satellite- to be determined from relevant power calculations.

Achieved concentration at all dose levels and homogeneity and stability at high and low doses.

Continuous for 28 days for main study, satellite, and recovery groups.

Daily detailed observations, days 1-29 main study, recovery and satellite animals; recovery and satellite animals in weeks 5 and 6 . Daily cage-side observations.

All animals daily, days 1-29. Thereafter, satellite and recovery animals weekly: days 36 and 43 .

Monitored continuously throughout the study.

All main study animals in week 4 (including landing foot splay, grip strength, and tail-flick) and recovery animals in week 6 .

All main study animals in week 4 and recovery animals in week 6 .

Urine samples for clinical chemistry from main study animals during week 4 and from recovery animals during week 6. Blood samples for hematology and clinical chemistry from all main study and recovery animals at scheduled termination. Satellite groups not evaluated.

Main study and recovery—guideline-specified tissues (based on OECD 407 [OECD, 1995] and U.S. EPA OPPTS 870.3100 [U.S. EPA, 1998a]), and any macroscopic abnormalities submitted from all rats. Guideline-specified organs weighed.

Neuropathology satellites-The following tissues will be taken from all rats sacrificed by perfusion fixation and preserved in an appropriate fixative: brain, eye (with optic nerve and retina), ${ }^{*}$ spinal cord (including cervical and lumbar swellings), spinal nerve roots (dorsal and ventral root fibers) at cervical swelling, spinal nerve roots (dorsal and ventral root fibers) at lumbar swelling, dorsal root ganglia at cervical swelling, dorsal root ganglia at lumbar swelling, proximal sciatic nerve, ${ }^{*}$ proximal tibial nerve, ${ }^{*}$ distal tibial nerve (tibial nerve calf muscle branches), ${ }^{*}$ and gastrocnemius muscle. ${ }^{*}\left({ }^{*}=\right.$ left for processing, right for store.)

\section{Proposed Design of the Core 90-Day Toxicity Study in the Dog}

- The proposed 90-day dog study is pivotal in the assessment of subchronic toxicity, and is used to provide information on relevance to human exposure durations from 1 day up to 6 months either alone or in conjunction with the 28-day rat study.
- Dose levels and study design will be influenced by the quantitative and qualitative outcome of the 28-day study in the rat and by the outcome of a preliminary study in the dog, and will be supported by relevant ADME data. Satellite animals may be added to the standard study design to assess specific effects (such as neuropathology) if the need is indicated from the outcome of preceding studies. 


\section{Summary of Study Specification-Core 90-Day Toxicity Study in the Dog}

Groups: One control and typically three test.

Number of animals: $\quad 6 /$ sex/group.

Dose administration: Once daily for 90 days by capsule or diet.

Analysis of dose preparations:

Clinical observations:

None in capsule (assumes neat test substance in capsule) or analysis of all batches of diet.

Veterinary examinations:

Pen-side observations three times daily. Detailed observations weekly.

Detailed clinical examination (including cardiac and pulmonary auscultation and body temperature) of all dogs prestudy and prior to termination.

Body weights: Weekly (for 2 weeks prestudy), on day 1, and weekly thereafter.

Food consumption: Daily (for 2 weeks prestudy and throughout the study).

Ophthalmoscopy: All dogs prestudy and prior to termination.

Physiological

measurements:

Lead II electrocardiogram and direct blood pressure recorded from all dogs prestudy, and then prior to and at one time point postdosing on day 1 and in weeks 7 and 13 .

Toxicokinetics:

Blood samples from all test group dogs at typically 6 time points after dosing on day 1 and in weeks 7 and 13.

Clinical pathology:

Blood samples for hematology and clinical chemistry taken from all dogs prestudy and in week 13. Urine samples (by catheterization) collected from all dogs prestudy and in week 13 .

Pathology:

Detailed examination post-mortem and tissues submitted from decedents and all animals at termination. Selected organs weighed from all animals at scheduled termination. All abnormalities, all submitted tissues from all animals processed and examined. (Based on OECD 409 [OECD, 1998] and U.S. EPA OPPTS 870.3150 [U.S. EPA, 1998b].)

Data evaluation:

Report:

Relevant data will be analyzed statistically using relevant statistical approaches.

Full quality-assured report with individual animal data supplement.

\section{Proposed Design of the Single-Dose Toxicity Study} in the Rat/Dog

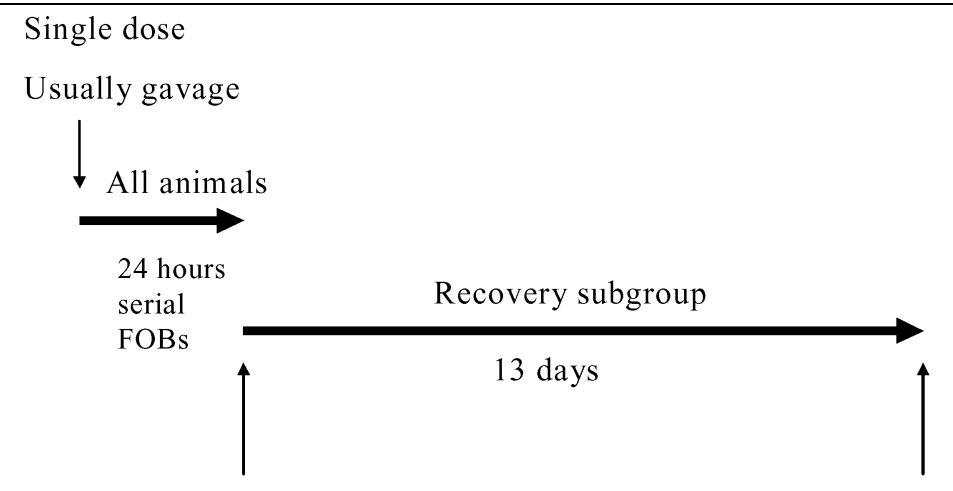

24 hour sacrifice

Recovery sacrifice

Full evaluation

Full evaluation

- The single-dose study is intended to provide data that may be used to refine risk assessments relevant to human exposure durations of up to 1 day, as well as to intermittent exposures. The study design is based on that proposed in setting an acute reference dose (Solecki, 2001).

- Dose levels and study design will be influenced by the quantitative and qualitative outcome of the 28-day rat study and 90-day dog study, and will be supported by relevant ADME data.

- The numbers of experimental animals used for the study are scientifically based on power calculations as described for the 28-day rat study or, for the dog study, six dogs/sex/dose level will be used. 


\section{Summary of Study Specification-Single-Dose Toxicity Study in the Rat/Dog}

Groups:

Number of animals:

Analysis of dosing

preparation:

Dose administration:

Clinical observations:

Body weights:

Food consumption:

Functional observational battery:

Motor activity:

Clinical pathology:

Pathology:
One control and up to three test.

To be determined from relevant power calculations (see above); equal numbers of animals sacrificed at 24 hours and at the end of the 14-day recovery phase.

Achieved concentration at all dose levels and homogeneity and stability at high and low doses (gavage dosing).

Single dose by oral gavage (rat) or by capsule (dog).

Daily detailed observations at time of peak effect and at $0.5,1,2,4$, and 24 hours after dosing; daily thereafter.

All animals daily for the first week, then prior to sacrifice of the recovery subgroup.

Monitored continuously throughout the study.

All animals at time of peak effect, prior to sacrifice at 24 hours, or after the recovery period.

All animals at time of peak effect, prior to sacrifice at 24 hours, or after the recovery period.

Urine samples for clinical chemistry from interim sacrifice animals and, if effects observed, again at the end of the recovery period. Blood samples for hematology and clinical chemistry from all animals at scheduled termination.

Range of tissues weighed and examined histopathologically for all animals at scheduled termination. Any macroscopic abnormalities submitted from all animals. Requirement based on OECD 407 (OECD, 1995) and U.S. EPA OPPTS 870.3100 for the rat (U.S. EPA, 1998a), and OECD 409 (OECD, 1998) and US EPA 870.3150 for the dog (U.S. EPA, 1998b).

\section{Proposed Design of the 24-Month Combined Chronic Toxicity/Carcinogenicity Study in the Rat}

The 24-month combined chronic toxicity/carcinogenicity study in the rat includes an assessment of chronic toxicity at 12 months. Conduct of this study (if indicated from the duration of human exposure) would lead to a waiver of the need to conduct a separate 12-month chronic toxicity study in this species.

\section{Summary of Study Specification-24-Month Rat Combined Chronic Toxicity/Carcinogenicity Study}

Groups:

Number of animals:

Analysis of diets:

Dose administration:

Clinical observations:

Ophthalmoscopy:

Body weights:

Food consumption:

Functional observational battery:

Motor activity:

Clinical pathology:

Pathology:
One control and up to five test.

50 males and 50 females for the main study, plus 10 males and 10 females for interim sacrifice at 12 months.

Achieved concentration at all dose levels and homogeneity and stability at high and low doses.

Continuous in the diet for up to 24 months.

Twice daily cage-side observations. Detailed observations recorded weekly—all study animals.

All animals examined pre-study, and control and high dose for main study and recovery groups examined prior to termination.

All animals weekly pre-study and throughout the study.

Monitored continuously throughout the study.

Interim sacrifice animals in week 52, and main study animals in weeks 14, 27, 53, 79 and prior to termination.

Interim sacrifice animals in week 52, and main study animals in weeks 14, 27, 53, 79 and prior to termination.

Urine samples for clinical chemistry from main study animals in weeks 14, 27, 53, 79 and at termination. Blood samples for hematology and clinical chemistry from all main study animals in weeks 14, 27, 53, 79 and at termination.

All animals, guideline-specified tissues (based on OECD 453 [OECD, 1981] and U.S. EPA OPPTS 870.4300 [U.S. EPA, 1998d]) and any macroscopic abnormalities submitted from all rats. Guideline-specified organs weighed. 